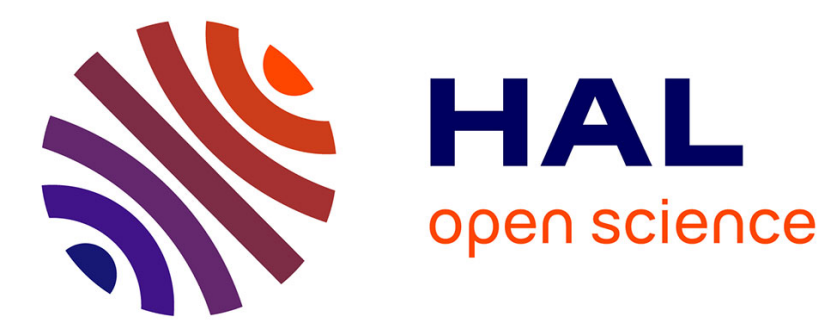

\title{
Obstructions to the existence of monotone Lagrangian embeddings into cotangent bundles of manifolds fibered over the circle
}

Agnès Gadbled

\section{- To cite this version:}

Agnès Gadbled. Obstructions to the existence of monotone Lagrangian embeddings into cotangent bundles of manifolds fibered over the circle. 2008. hal-00333176

\section{HAL Id: hal-00333176 \\ https://hal.science/hal-00333176}

Preprint submitted on 22 Oct 2008

HAL is a multi-disciplinary open access archive for the deposit and dissemination of scientific research documents, whether they are published or not. The documents may come from teaching and research institutions in France or abroad, or from public or private research centers.
L'archive ouverte pluridisciplinaire HAL, est destinée au dépôt et à la diffusion de documents scientifiques de niveau recherche, publiés ou non, émanant des établissements d'enseignement et de recherche français ou étrangers, des laboratoires publics ou privés. 


\title{
Obstructions to the existence of monotone Lagrangian embeddings into cotangent bundles of manifolds fibered over the circle
}

\author{
Agnès GADBLED *
}

22nd October 2008

\begin{abstract}
We extend the constructions and results of Damian [ical obstructions to the existence of closed monotone Lagrangian embeddings into the cotangent bundle of a space which is the the total space of a fibration over the circle.
\end{abstract}

\section{Introduction}

Let $M$ be a closed manifold and $\pi: T^{*} M \rightarrow M$ its cotangent bundle. Denote by $\lambda_{M}$ the Liouville one-form of $M$ and $\omega_{M}=d \lambda_{M}$ the canonical symplectic form on $T^{*} M$.

We are interested in compact Lagrangian submanifolds in the cotangent bundle $T^{*} M$. Only a few types of examples are known:

1. the zero section or more generally the graph $L_{f}$ of a function $f: M \rightarrow \mathbb{R}$;

2. any Hamiltonian image of $L_{f}$ (i.e. $L=\varphi_{1}\left(L_{f}\right)$ where $\left(\varphi_{t}\right)$ is a Hamiltonian isotopy);

3. any image of $L_{f}$ by a symplectic isotopy (as in 2 but with $\left(\varphi_{t}\right)$ a symplectic isotopy);

4. the "local" Lagrangian submanifolds: any Lagrangian submanifold of $\mathbb{C}^{n}$ can be embedded in a Darboux chart $U \stackrel{\sim}{\rightarrow} \mathbb{C}^{n}$ of $T^{*} M$.

Note that the two first types of examples have the additional property of being exact (that is, the restriction of the Liouville one-form on the Lagrangian submanifold is exact). It is conjectured (see 11) that the examples (1) and (2) are the only possible examples of exact Lagrangian submanifolds in $T^{*} M$ and much work has been done to prove this conjecture. It has been proved for $L=M=S^{2}$ (Hind [9]) but in general, we know only topological restrictions on the exact Lagrangian embeddings $i: L \rightarrow T^{*} M$ of a closed manifold $L$. In the following, $f: L \rightarrow M$ denotes the composition $\pi \circ i$.

*MSC classification: 57R17, 57R58, 57R70, 53D12.

Keywords: Lagrangian embeddings, Floer homology, Novikov homology. 
1. (Audin [2]) If $L$ and $M$ are orientable, then $\chi(L)=\operatorname{deg}(f)^{2} \chi(M)$ and the same is true modulo 4 if $L$ and $M$ are not orientable.

2. (Lalonde and Sikorav [10]) The index $\left[\pi_{1}(M): f_{\star}\left(\pi_{1}(L)\right)\right]$ is finite.

3. (Viterbo [24]) If $M$ is simply connected, then $L$ cannot be an EilenbergMacLane space.

4. (Fukaya, Seidel and Smith [8], Nadler [14, see also Buhovski [4]) If $M$ is simply connected and $L$ is spin with zero Maslov class, then the projection $f$ has degree \pm 1 , and induces an isomorphism $H^{\star}(L, K) \simeq H^{\star}(M, K)$ for any field $K$ of characteristic not equal to 2 .

5. (Ritter [21]) If $M$ is simply connected, then $\pi^{\star}: H^{2}(M) \rightarrow H^{2}(L)$ is injective and the image of $f_{\star}: \pi_{2}(L) \rightarrow \pi_{2}(M)$ has finite index.

6. (Damian [5]) If $M$ has dimension $n \geq 3$ and is the total space of a fibration over $\mathbb{S}^{1}$, we have:

a) For any finite presentation $\left\langle g_{1}, g_{2}, \ldots, g_{p} \mid r_{1}, r_{2}, \ldots, r_{q}\right\rangle$ of the fundamental group $\pi_{1}(L), p-q \leq 1$.

b) The fundamental group $\pi_{1}(L)$ is not isomorphic to the free product $G_{1} * G_{2}$ of two non-trivial groups.

The idea of the proof of Damian is the following. On the one hand, if $M$ is the total space of a fibration $p: M \rightarrow \mathbb{S}^{1}$ over the circle, then one can use the pull-back $\alpha=p^{\star} d \theta$ of the one-form $d \theta$ on $\mathbb{S}^{1}$ to displace an exact Lagrangian submanifold $L$ of $T^{*} M$ from itself by a symplectic isotopy (defined by $\left.\varphi_{t}(q, p)=(q, p+t \alpha)\right)$.

On the other hand, given a symplectic isotopy $\left(\varphi_{t}\right)$, Damian has constructed a Floer-type complex $C\left(L, \varphi_{t}\right)$ spanned by the intersection points of $L$ and $\varphi_{1}(L)$, with coefficients in a Novikov ring associated to $\pi_{1}(L)$ and endowed with a differential which is an analogue in the Lagrangian Floer theory of the Morse-Novikov differential. The homology of this complex only depends on the flux $\left[\varphi_{1}^{\star} \lambda_{M}-\lambda_{M}\right]=u$ of $\left(\varphi_{t}\right)$. It is called the Floer-Novikov homology of $L$ and denoted $F H(L, u)$.

Damian has proved that this homology $F H(L, u)$ is isomorphic to the Novikov homology $H_{\star}\left(L, f^{\star} u\right)$ of $L$. The Novikov homology of $L$ must then be trivial when $\varphi_{t}$ is the isotopy induced by $\alpha$, that is when $u=[\alpha]$, and this gives the obstructions on the fundamental group of $L$.

Here we are interested in the more general case of monotone Lagrangian submanifolds of $T^{*} M$. In the usual sense, a Lagrangian submanifold $L$ is monotone (on the disks) if there exists a non-negative constant $K_{L}$ such that:

$$
\text { for all } u \in \pi_{2}\left(T^{*} M, L\right), \int_{u} \omega_{M}=K_{L} \mu_{L}(u)
$$

where $\mu_{L}$ denotes the Maslov class of $L$ in $T^{*} M$.

For instance, any local Lagrangian submanifold which is monotone in $\mathbb{C}^{n}$ is also monotone in $T^{*} M$ (see Remark 1.2 (iii)).

We would like to know if there are also "global" monotone Lagrangian submanifolds in $T^{*} M$. It is possible to get topological obstructions on the monotone 
Lagrangian embeddings by extending the construction of the Floer-Novikov type complex of Damian to the monotone case. In order to carry out this construction, we need a stronger monotonicity assumption:

Definition 1.1. A Lagrangian submanifold of $T^{*} M$ is said to be monotone on the loops if there exists a non-negative constant $k_{L}$ such that:

$$
\text { for all } \gamma \in \pi_{1}(L), \int_{\gamma} \lambda_{M}=k_{L} \mu_{L}(\gamma)
$$

where $\mu_{L}$ denotes the Maslov class of $L$ in $T^{*} M$.

Remarks 1.2. (i) We recover the exact case when $k_{L}=0$.

(ii) If $L$ is a Lagrangian submanifold of $T^{*} M$ which is monotone on the loops, then it is monotone in the usual sense (i.e. on the disks) with the same constant $k_{L}=K_{L}$.

(iii) Note that the converse of (iii) is not necessarily true in general. It is true for instance if $M$ is simply connected.

(iv) This definition of monotone Lagrangian submanifold was already used by Polterovich [20 for Lagrangian submanifolds of $\mathbb{C}^{n}$, but in this case, it coincides with the usual definition.

(v) This assumption is necessary to bound from above the energy of solutions having the same Maslov index (see Section 3.5).

Notation 1.3. If $L$ is a Lagrangian submanifold in $T^{*} M$ which is monotone on the disks, we will call Maslov number of $L$ and denote $N_{L}$ the non-negative generator of the subgroup $\left\langle\mu_{L}, \pi_{2}(L)\right\rangle$ of $\mathbb{Z}$.

As in the exact case, we will use a suitable version of Floer-Novikov homology. The differences are the following:

(i) There is no action functional, so we will have to work with the action oneform (see Remark 3.11).

(ii) The fact that $\left[\pi_{1}(M): f_{\star}\left(\pi_{1}(L)\right)\right]$ is finite does not hold in the monotone case without further assumption on the Maslov class of $L$. For instance, for any local monotone Lagrangian submanifold in a Darboux chart, $f_{\star}$ : $\pi_{1}(L) \rightarrow \pi_{1}(M)$ is trivial and the index is not finite unless $\pi_{1}(M)$ is finite.

(iii) In the monotone case, we have to take into account the bubbling of $J$ holomorphic disks. This makes the definition of Floer-Novikov homology more intricate.

(iv) There are also differences on more technical points. For instance, in the proof of invariance, we cannot use an extension of a symplectic isotopy of $T^{*} L$ to $T^{*} M$ as in the exact case.

(v) In the monotone case, the Floer homology is not always isomorphic to the Novikov homology $H_{\star}\left(L, f^{\star} u\right)$. We will prove that it is the limit of a spectral sequence (see Theorems 1.4 and 4.7, this is a "Novikov version" of the spectral sequence described by Biran in [3] for the usual Lagrangian Floer homology). 
Theorem 1.4. Let $u$ be an element of $H^{1}(M)$. Assume that the Lagrangian submanifold $L$ is monotone on the loops, and of Maslov number $N_{L} \geq 2$. There exists a spectral sequence $\left\{E_{r}^{p, q}, d_{r}\right\}$ satisfying the following properties:

(1) $E_{0}^{p, q}=C_{p+q-p N_{L}}\left(L, f^{\star} u\right) \otimes A^{p N_{L}}$ and $d_{0}=\partial_{0} \otimes 1$, where $\partial_{0}$ is the MorseNovikov differential;

(2) $E_{1}^{p, q}=H_{p+q-p N_{L}}\left(L, f^{\star} u\right) \otimes A^{p N_{L}}$ and $d_{1}=\left[\partial_{1}\right] \otimes \tau$ where

$$
\left[\partial_{1}\right]: H_{p+q-p N_{L}}\left(L, f^{\star} u\right) \rightarrow H_{p+1+q-(p+1) N_{L}}\left(L, f^{\star} u\right)
$$

(3) $\left\{E_{r}^{p, q}, d_{r}\right\}$ collapses at the page $\kappa+1$, where $\kappa=\left[\frac{\operatorname{dim}(L)+1}{N_{L}}\right]$ and the spectral sequence converges to $F H(L, u)$, i.e.

$$
\bigoplus_{p+q=\ell} E_{\infty}^{p, q} \cong F H^{\ell\left(\bmod N_{L}\right)}(L, u) .
$$

Here $A$ is the $\Lambda_{f \star}$-module $A=\Lambda_{f^{\star} u}\left[T, T^{-1}\right]$ of Laurent polynomials with coefficients in $\Lambda_{f^{\star} u}$ (see section 2) and $\tau_{i}: A \rightarrow A$ is the multiplication by $T^{i}$. The degree of $T$ is equal to $N_{L}$ and $A^{p N_{L}}=\Lambda_{f \star u} T^{p}$.

In particular, when $N_{L} \geq \operatorname{dim}(M)+2$, the spectral sequence above collapses at the first page and the Floer-Novikov homology is equal to the Novikov homology $H\left(L, f^{\star} u\right)$.

Using the techniques of Damian, we prove:

Theorem 1.5. Let $M$ be a closed manifold which is the total space of a fibration $p: M \rightarrow \mathbb{S}^{1}$ on the circle. Let $L$ be a Lagrangian submanifold of $T^{*} M$ which is monotone on the loops. Assume either that $N_{L} \geq \operatorname{dim}(M)+1$ or $\left(N_{L}=\operatorname{dim}(M)\right.$ and $\left[\pi_{1}(M): \pi_{1}(L)\right]$ is finite). We have:

(i) If $\left\langle g_{1}, g_{2}, \ldots, g_{p} \mid r_{1}, r_{2}, \ldots, r_{q}\right\rangle$ is a finite presentation of $\pi_{1}(L)$, then

$$
p-q \leq 1
$$

(ii) The fundamental group $\pi_{1}(L)$ is not isomorphic to a free product $G_{1} \star G_{2}$ of two non trivial groups.

This theorem will be proved in section 4

Acknowledgements. I would like to thank Michèle Audin and Mihai Damian for suggesting me to work on this extension of Floer homology, for valuable discussions around this subject and for their careful reading. I would also like to thank Jean-Claude Sikorav for his numerous remarks on the work which has given rise to this article.

\section{Novikov theory}

Let us recall the definition of Novikov homology (15), for a more detailed study, see [22]) and the results of Damian [5]. 
Let $L$ be a closed manifold and $u \in H^{1}(L, \mathbb{R})$. Denote by $\Lambda$ the ring $\mathbb{Z} / 2\left[\pi_{1}(L)\right]$ and by $\widehat{\Lambda}=\mathbb{Z} / 2\left[\left[\pi_{1}(L)\right]\right]$ the group of formal series.

Let $\Lambda_{u}$ be the completed ring of series

$$
\Lambda_{u}=\left\{\sum n_{i} g_{i} \in \widehat{\Lambda} \mid g_{i} \in \pi_{1}(L), n_{i} \in \mathbb{Z} / 2, u\left(g_{i}\right) \rightarrow+\infty\right\}
$$

where $u\left(g_{i}\right) \rightarrow+\infty$ means here that for all $A>0$, the set

$$
\left\{g_{i} \mid n_{i} \neq 0 \text { and } u\left(g_{i}\right)<A\right\}
$$

is finite.

Definition 2.1. Let $C(\widetilde{L})$ be the free $\Lambda$-complex spanned by fixed lifts of the cells of a $C W$-decomposition of $L$ to the universal cover $\widetilde{L}$ of $L$ and

$$
C(L, u)=\Lambda_{u} \otimes_{\Lambda} C(\widetilde{L}) .
$$

The homology of this complex $C(L, u)$ is the Novikov homology $H(L, u)$.

Definition 2.2 (Morse-Novikov homology). Let $\alpha$ be a closed generic oneform in the class of $u \in H^{1}(L, \mathbb{R})$ and $\xi$ be the gradient of $\alpha$ with respect to some generic metric on L. For every critical point $c$ of $\alpha$, fix a lift $\widetilde{c}$ of $c$ in the universal cover $\widetilde{L}$.

Let $C(\alpha, \xi)$ be the $\Lambda_{u}$-complex spanned by the zeros of $\alpha$ and whose differential is such that if $c$ and $d$ are zeros of index difference equal to 1 then their incidence number is the algebraic number of flow lines that joins $c$ to $d$ and lifts to a path in $\widetilde{L}$ from $g_{i} \widetilde{c}$ to $\widetilde{d}$.

By generic, we mean here that $\alpha$ has Morse-type singularities and $\xi$ satisfies the Morse-Smale condition.

Theorem 2.3 (Novikov [15, Latour [1], see also Pazhitnov 19]). For any generic pair $(\alpha, \xi)$, the homology of this complex is isomorphic to $H(L, u)$.

Remark 2.4. In these two descriptions of the Novikov homology, one could replace $\widetilde{L}$ by any integration covering $\bar{L}$ of $L$ (i.e. such that the pull-back of $u$ is zero). We will use this in our comparison between the Floer-Novikov homology and the Novikov homology of $L$ in Section 1 .

Let us end this section by recalling results proved in [5]:

Proposition 2.5 (Damian [5], Sikorav [22]). Let $L$ be a closed manifold and $u \in H^{1}(L, \mathbb{R})$.

a) Let $\left\langle g_{1}, g_{2}, \ldots, g_{p} \mid r_{1}, r_{2}, \ldots, r_{q}\right\rangle$ be a presentation of the fundamental group $\pi_{1}(L)$ which satisfies $p-q \geq 2$. If $u \neq 0$, then $H_{1}(L, u) \neq 0$.

b) Suppose that $\pi_{1}(L)=G_{1} * G_{2}$ is a free product of two groups, none of them being trivial. If $u \neq 0$, then $H_{1}(L, u) \neq 0$. 


\section{The Floer type complex}

Let $i: L \hookrightarrow T^{*} M$ be a Lagrangian embedding of a closed manifold $L$. Assume that the image of $L$ in $T^{*} M$ is monotone on the loops. Let $\left(\varphi_{t}\right)$ be a symplectic isotopy of $T^{*} M$ and denote by $L_{t}$ the image of $L$ by $\left(\varphi_{t}\right)$.

Remark 3.1. As they are the images of $L$ by symplectomorphisms, the Lagrangian submanifolds $L_{t}$ are not necessarily monotone on the loops but they are monotone on the disks. We can therefore apply to them all the results concerning monotone (in the usual sense) Lagrangian submanifolds.

Let $u \in H^{1}(M ; \mathbb{R})$ denote the flux (or Calabi invariant) of $\left(\varphi_{t}\right)$, that is the class:

$$
\operatorname{Cal}\left(\varphi_{t}\right)=\left[\varphi_{1}^{\star} \lambda_{M}-\lambda_{M}\right] \in H^{1}\left(T^{*} M, \mathbb{R}\right) \simeq H^{1}(M, \mathbb{R}) .
$$

The results proved in Sections 3.1 to 3.5 are used in Section 3.5 to define a $\Lambda_{u}$-complex $C\left(\bar{L}_{0}, \bar{L}_{1}\right)$ spanned by the intersection points of $L_{0}=\varphi_{0}(L)$ and $L_{1}=\varphi_{1}(L)$. We prove in Section 3.6 that this complex depends only on $L$ and $u=\operatorname{Cal}\left(\varphi_{t}\right)$. We then explain in Section 3.7 that these results can be used to define a free complex over the Novikov ring $\Lambda_{f^{\star} u}$ spanned by $L \cap \varphi_{1}(L)$ whose homology only depend on $L$ and $u$.

\subsection{Preliminary results}

Let us first notice that it is possible to restrict our symplectic isotopies to isotopies of the following type:

Lemma 3.2. There exists a symplectic isotopy $\left(\psi_{t}\right)$ on $T^{*} M$ such that $\psi_{1 \mid L}=$ $\varphi_{1 \mid L}$ which is spanned by $\alpha+d H_{t}$, where $\alpha$ is a closed one-form in $u$ and $H: T^{*} M \times[0,1] \rightarrow \mathbb{R}$ has compact support.

Proof. As in the proof of [5, Lemma 3.2] (which does not use the exactness assumption on $L$ ), consider a family of one-forms $\alpha_{t}$ on $M$ in the class of $\varphi_{t}^{\star} \lambda_{M}-$ $\lambda_{M}$. Note that the composition of $\left(\varphi_{t}\right)$ and of the symplectic isotopy spanned by $-\alpha_{t}$ is a compactly supported Hamiltonian isotopy $\chi_{t}$. The isotopy $\left(\psi_{t}\right)$ can then be defined as the composition of $\chi_{t}$ with the symplectic isotopy spanned by $\alpha_{1}$.

We will also require in the construction and applications that the induced map $f_{\star}: \pi_{1}(L) \rightarrow \pi_{1}(M)$ is surjective. It is enough to suppose that the index $\left[\pi_{1}(M): f_{\star}\left(\pi_{1}(L)\right)\right]$ is finite:

Remark 3.3. If $f_{\star}: \pi_{1}(L) \rightarrow \pi_{1}(M)$ is not surjective, let $M_{1}$ be the covering of $M$ induced by the subgroup $f_{\star}\left(\pi_{1}(L)\right)$ of $\pi_{1}(M)$. The manifold $L$ can be lifted as a Lagrangian submanifold of $T^{*} M_{1}$ which is monotone on the loops (and which has the same Maslov class).

If $\left[\pi_{1}(M): f_{\star}\left(\pi_{1}(L)\right)\right]$ is finite, then $M_{1}$ is closed and the conclusion of Theorem 1.5 in $T^{*} M$ is then just a consequence of the same theorem for $T^{*} M_{1}$ where the surjectivity condition is satisfied.

Although the index $\left[\pi_{1}(M): f_{\star}\left(\pi_{1}(L)\right)\right]$ is always finite in the exact case (Lalonde and Sikorav, 10, Theorem 1 a)]), this assumption is not always fulfilled in the monotone case. However, the index is necessarily finite for monotone Lagrangian submanifolds if the Maslov number of $L$ is large enough: 
Proposition 3.4. Let $L$ be a Lagrangian submanifold of $T^{*} M$ which is monotone (on the disks). If $N_{L} \geq \operatorname{dim}(M)+1$, then the index of $\pi_{1}(L)$ in $\pi_{1}(M)$ is finite.

Proof. If $\pi_{1}(L) \rightarrow \pi_{1}(M)$ is not surjective, consider again the covering $M_{1}$ of $M$ induced by the subgroup $f_{\star}\left(\pi_{1}(L)\right)$ of $\pi_{1}(M)$ and the lift of $L$ into $T^{*} M_{1}$.

If the covering group of $M_{1} \rightarrow M$ is infinite, then $M_{1}$ is open and the Lagrangian submanifold $L$ can be displaced from itself by a Hamiltonian isotopy (see [10, Proposition 1]) so that the usual Floer homology $H F(L, L)$ (with $\mathbb{Z} / 2$ coefficients) must be trivial. But if the Maslov number of $L$ is greater than $\operatorname{dim}(M)+2$, we know by Oh's theorem ([18, Theorem II (i)]) that this homology is isomorphic to the usual cohomology $H^{\star}(L, \mathbb{Z} / 2)$ of $L$ and this is in contradiction with the vanishing of $H F(L, L)$.

If $N_{L}=\operatorname{dim}(M)+1$, then by [18, Theorem II (ii)],

$$
H F(L, L) \approx \bigoplus_{i=0}^{\operatorname{dim}(M)} H^{i}(L, \mathbb{Z} / 2) \text { or } \bigoplus_{i=1}^{\operatorname{dim}(M)-1} H^{i}(L, \mathbb{Z} / 2)
$$

and this also leads to a contradiction unless $L$ is a $\mathbb{Z} / 2$-homology sphere.

However, if $L$ is a $\mathbb{Z} / 2$-homology sphere, then $H^{1}(L, \mathbb{R})=0$ and in particular $L$ is exact so that we can directly apply the result of Lalonde and Sikorav to see that the index is finite.

Remark 3.5. We cannot expect to remove the assumption $N_{L} \geq \operatorname{dim}(M)+1$ in Proposition 3.4. Indeed, Polterovich proved in 20] (see also Audin 20 for the construction) that for every two integer numbers $2 \leq k \leq n$, there exists a compact manifold $L_{n, k}$ which admits a monotone Lagrangian embedding in $\mathbb{C}^{n}$ (and consequently "local" monotone Lagrangian submanifolds in any cotangent bundle) with Maslov number equal to $k$ such that:

a) $L_{n, n}=\mathbb{S}^{n-1} \times \mathbb{S}^{1} / \tau_{n-1} \times \tau_{1}$ where $\tau_{j}: \mathbb{S}^{j} \rightarrow \mathbb{S}^{j}$ is the antipodal involution;

b) $L_{n, k}=L_{k, k} \times \mathbb{S}^{n-k}$ where $k<n$.

Remark 3.6. Thanks to Remark 3.3 and Proposition 3.4, it is enough to prove Theorem 1.5 when the induced map

$$
f_{\star}: \pi_{1}(L) \longrightarrow \pi_{1}(M)
$$

is surjective. From now on, we will always suppose that $f_{\star}$ is surjective.

Thanks to the surjectivity assumption on $f_{\star}$, we can consider a connected covering of the Lagrangian submanifold $L$ in the cotangent bundle of the universal cover of $M$ :

Lemma 3.7. Let $\widetilde{M} \rightarrow M$ be the universal covering of $M$ and $\widetilde{p}: T^{*} \widetilde{M} \rightarrow T^{*} M$ be the induced covering on the cotangent bundles. Denote by $y \mapsto y^{g}$ the right action of $\pi_{1}(M)$ on $T^{*} \widetilde{M}$.

Let $\bar{L} \rightarrow L$ be the pull-back of the covering $T^{*} \widetilde{M} \rightarrow T^{*} M$ by the embedding $i: L \rightarrow T^{*} M$. Then:

(i) $\bar{L}$ is path-connected if and only if the map $f_{\star}: \pi_{1}(L) \rightarrow \pi_{1}(M)$ is surjective. 
(ii) The covering $\bar{L} \rightarrow L$ corresponds to the covering of $L$ associated with the subgroup $K=\operatorname{ker}\left(f_{\star}\right)$ of $\pi_{1}(L)$.

(iii) The map $\widetilde{\imath}: \bar{L} \rightarrow T^{*} \widetilde{M}$ is a monotone Lagrangian embedding and for all $g \in \pi_{1}(M), \widetilde{x} \in \bar{L}$,

$$
\widetilde{\imath}\left(\widetilde{x}^{g}\right)=(\widetilde{\imath}(\widetilde{x}))^{g}
$$

The proof of this lemma uses only basic algebraic topology and can be found in the appendix.

Lemma 3.8. Let $\left(\varphi_{t}\right)$ be the symplectic isotopy generated by $\alpha+d H_{t}$. Then this isotopy lifts to a Hamiltonian isotopy $\left(\widetilde{\varphi}_{t}\right)$ on $T^{*} \widetilde{M}$.

Moreover, if $L_{t}=\varphi_{t}(L)$ and $\bar{L}_{t}=\widetilde{\varphi}_{t}(\bar{L})$, then

$$
\bar{L} \cap \bar{L}_{1}=\bigcup_{x \in L \cap L_{1}} \tilde{p}^{-1}(x) .
$$

Proof. As in the proof of [5, Lemma 3.6], which does not involve any assumption on the exactness/monotonicity of $L$, we can define the isotopy $\left(\widetilde{\varphi}_{t}\right)$ as the isotopy spanned by the pullback of $\alpha+d H_{t}$ to $T^{*} \widetilde{M}$.

\subsection{The action one-form}

In this section, $L$ is assumed to be a closed Lagrangian submanifold of $T^{*} M$. It is also assumed that $L$ is monotone on the loops and that $f_{\star}: \pi_{1}(L) \rightarrow \pi_{1}(M)$ is surjective.

Let $\left(\varphi_{t}\right)$ be a symplectic isotopy as in Lemma 3.2. Denote by $u \in H^{1}(M ; \mathbb{R})$ its flux.

If $L_{t}=\varphi_{t}(L)$, let $\Omega\left(L_{0}, L_{1}\right)$ be the space of paths from $L_{0}$ to $L_{1}$ :

$$
\Omega\left(L_{0}, L_{1}\right)=\left\{z \in C^{\infty}\left([0,1] ; T^{*} M\right) \mid z(i) \in L_{i}, i=0,1\right\} .
$$

We define a one-form on $\Omega\left(L_{0}, L_{1}\right)$ by:

$$
\nu_{z}(V)=\int_{0}^{1} \omega_{M}\left(z^{\prime}(t), V(t)\right) d t
$$

The zeros of $\nu$ are the constant maps, that is, the intersection points of $L_{0}$ and $L_{1}$.

The integral of $\nu$ on a loop involves the one-form $u$, as in the exact case, but also the monotonicity constant $k_{L}$ of $L$ :

Proposition 3.9. Let $\gamma: \mathbb{S}^{1} \rightarrow \Omega\left(L_{0}, L_{1}\right)$ be a loop. Then

$$
\begin{aligned}
\int_{\gamma} \nu & =\lambda_{M}\left(\gamma_{0}\right)-\lambda_{M}\left(\varphi_{1}^{-1}\left(\gamma_{1}\right)\right)-u\left(\gamma_{0}\right) \\
& =k_{L}\left(\mu_{L_{0}}\left(\gamma_{0}\right)-\mu_{L_{1}}\left(\gamma_{1}\right)\right)-u\left(\gamma_{0}\right)
\end{aligned}
$$

denoting $\gamma_{i}=\gamma\left(\mathbb{S}^{1} \times\{i\}\right)$ for $i=0,1$. 
Proof. Considering the loop $\gamma$ as a map $\gamma: \mathbb{S}^{1} \times[0,1] \rightarrow T^{*} M$, we get

$$
\int_{\gamma} \nu=\int_{\mathbb{S}^{1}} \nu\left(\frac{\partial \gamma}{\partial s}\right) d s=\int_{\mathbb{S}^{1}} \int_{0}^{1} \omega_{M}\left(\frac{\partial \gamma}{\partial t}, \frac{\partial \gamma}{\partial s}\right) d t d s=-\int_{\gamma\left(\mathbb{S}^{1} \times[0,1]\right)} \omega_{M} .
$$

Then, by the Stokes formula,

$$
\begin{aligned}
\int_{\gamma} \nu & =\int_{\gamma\left(\mathbb{S}^{1} \times\{0\}\right)} \lambda_{M}-\int_{\gamma\left(\mathbb{S}^{1} \times\{1\}\right)} \lambda_{M} \\
& =\int_{\gamma\left(\mathbb{S}^{1} \times\{0\}\right)} \lambda_{M}-\int_{\varphi_{1}^{-1}\left(\gamma\left(\mathbb{S}^{1} \times\{1\}\right)\right)} \varphi_{1}^{\star} \lambda_{M} \\
& =\int_{\gamma\left(\mathbb{S}^{1} \times\{0\}\right)} \lambda_{M}-\int_{\varphi_{1}^{-1}\left(\gamma\left(\mathbb{S}^{1} \times\{1\}\right)\right)} \lambda_{M}-\int_{\varphi_{1}^{-1}\left(\gamma\left(\mathbb{S}^{1} \times\{1\}\right)\right)}\left(\varphi_{1}^{\star} \lambda_{M}-\lambda_{M}\right) .
\end{aligned}
$$

As $\varphi_{1}^{\star} \lambda_{M}-\lambda_{M}$ is a closed one-form in the cohomology class $u=\operatorname{Cal}\left(\varphi_{t}\right)$, the third term is equal to:

$$
u\left(\varphi_{1}^{-1}\left(\gamma\left(\mathbb{S}^{1} \times\{1\}\right)\right)\right)=u\left(\gamma\left(\mathbb{S}^{1} \times\{0\}\right)\right) .
$$

So that

$$
\int_{\gamma} \nu=\lambda_{M}\left(\gamma_{0}\right)-\lambda_{M}\left(\varphi_{1}^{-1}\left(\gamma_{1}\right)\right)-u\left(\gamma_{0}\right) .
$$

We now use the monotonicity of $L$ to write:

$$
\begin{aligned}
\int_{\gamma} \nu & =k_{L}\left(\mu_{L}\left(\gamma_{0}\right)-\mu_{L}\left(\varphi_{1}^{-1}\left(\gamma_{1}\right)\right)\right)-u\left(\gamma_{0}\right) \\
& =k_{L}\left(\mu_{L_{0}}\left(\gamma_{0}\right)-\mu_{L_{1}}\left(\gamma_{1}\right)\right)-u\left(\gamma_{0}\right)
\end{aligned}
$$

since $\varphi_{1}$ is a symplectic isotopy.

Corollary 3.10. The action one-form $\nu$ is closed.

Proof. The formula (3) proves that $\int_{\gamma} \nu$ depends only on the homotopy class of $\gamma$ in $\Omega\left(L_{0}, L_{1}\right)$.

Remark 3.11. We could also lift $\nu$ to $\Omega\left(\bar{L}_{0}, \bar{L}_{1}\right)$ (as in the exact case, see []) but the one-form is not necessarily exact on this space. Nevertheless, we will be able to carry out the construction of the complex without needing a primitive of $\nu$.

\section{Alternative setting}

We can also define a one-form on $\Omega(L, L)$. This setting will be useful in the proof of Hamiltonian invariance (Section 3.6).

Let $X_{t}^{\alpha+d H_{t}}$ be the symplectic dual of $\alpha+d H_{t}$, defined by $\omega_{M}\left(\cdot, X_{t}^{\alpha+d H_{t}}\right)=$ $\left(\alpha+d H_{t}\right)(\cdot)$. Denote by $\left(\varphi_{t}\right)$ the isotopy spanned by $X_{t}^{\alpha+d H_{t}}$.

We can define a one-form $\widehat{\nu}$ on $\Omega(L, L)$ by:

$$
\widehat{\nu}_{z}(V)=\int_{0}^{1} \omega_{M}\left(z^{\prime}(t), V(t)\right)+\left(\alpha+d H_{t}\right)(V(t)) d t .
$$


The zeros of $\widehat{\nu}$ are the flow trajectories beginning on $L$ (at time 0 ) and ending on $L$ (at time 1 ).

If $\gamma: \mathbb{S}^{1} \rightarrow \Omega(L, L)$ is a loop in $\Omega(L, L)$, we have as in Proposition 3.9:

$$
\int_{\gamma} \widehat{\nu}=-\int_{\mathbb{S}^{1} \times[0,1]} \gamma^{\star} \omega_{M}+\int_{\mathbb{S}^{1}} \int_{0}^{1}\left(\alpha+d H_{t}\right)\left(\frac{\partial \gamma}{\partial s}\right) d t d s
$$

with

$$
\begin{aligned}
\int_{\mathbb{S}^{1}} \int_{0}^{1}\left(\alpha+d H_{t}\right)\left(\frac{\partial \gamma}{\partial s}\right) d t d s & =\int_{\mathbb{S}^{1}} \int_{0}^{1} \alpha\left(\frac{\partial \gamma}{\partial s}\right) d t d s=\int_{0}^{1} \int_{\gamma(\cdot, t)} \alpha d t \\
& =\int_{\gamma(\cdot, 0)} \alpha=u\left(\gamma_{0}\right)
\end{aligned}
$$

since $\int_{\gamma(\cdot, t)} \alpha$ does not depend on $t$. Thus, we have

$$
\begin{aligned}
\int_{\gamma} \widehat{\nu}= & \lambda_{M}\left(\gamma_{0}\right)-\lambda_{M}\left(\gamma_{1}\right)+u\left(\gamma_{0}\right) \\
& =k_{L}\left(\mu_{L}\left(\gamma_{0}\right)-\mu_{L}\left(\gamma_{1}\right)\right)+u\left(\gamma_{0}\right) .
\end{aligned}
$$

Remark 3.12. Note that these two settings are equivalent: if $\nu_{-}$is the oneform defined on $\Omega\left(L_{0}, \varphi^{-1}\left(L_{0}\right)\right)$ with the symplectic isotopy $\left(\varphi_{t}^{-1}\right)$, then the map $\Gamma(z)=\varphi_{t}^{-1}(z)$ is a 1 to 1 correspondence between $\Omega(L, L)$ and $\Omega\left(L_{0}, \varphi^{-1}\left(L_{0}\right)\right)$ and we have

$$
\Gamma^{\star} \nu_{-}=\widehat{\nu}
$$

(The fact that the correspondence uses $\varphi_{t}^{-1}$ instead of $\varphi_{t}$ explains the difference of signs between the relations (3) and (4) on the one hand, and (5) and (6) on the other hand.)

\subsection{The gradient}

Let $\left(J_{t}\right)$ be a family of almost complex structures on $T^{*} M$ that are compatible with $\omega_{M}$ and $\left(g_{t}\right)$ be the family of associated Riemannian metrics on $T^{*} M$.

We consider the trajectories of the opposite of the gradient of the one-form $\nu$ with respect to the induced metric on $\Omega\left(L_{0}, L_{1}\right)$. These are, as maps of two variables, solutions of the Cauchy-Riemann equation.

We define for a solution $v$ of the Cauchy-Riemann equation its energy

$$
E(v)=\int_{\mathbb{R} \times[0,1]}\left\|\frac{\partial v}{\partial s}\right\|^{2} d t d s .
$$

Denote then by $\mathcal{M}\left(L_{0}, L_{1}\right)$ the space of trajectories of finite energy:

$$
\mathcal{M}\left(L_{0}, L_{1}\right)=\left\{\begin{array}{l|l}
v \in \mathcal{C}^{\infty}\left(\mathbb{R} \times[0,1], T^{*} M\right) & \begin{array}{l}
\frac{\partial v}{\partial s}+J_{t}(v) \frac{\partial v}{\partial t}=0 \\
v(s, 0) \in L_{0} \text { and } v(s, 1) \in L_{1} \\
E(v)<\infty
\end{array}
\end{array}\right\} .
$$


In particular, if $v \in \mathcal{M}\left(L_{0}, L_{1}\right)$, then as in Proposition 3.9:

$$
E(v)=\int_{\mathbb{R} \times[0,1]} v^{\star} \omega_{M}=\int_{\mathbb{R} \times[0,1]} \omega_{M}\left(\frac{\partial v}{\partial s}, \frac{\partial v}{\partial t}\right) d t d s=-\int_{v} \nu .
$$

Denote also for $x, y \in L_{0} \cap L_{1}$ :

$$
\mathcal{M}(x, y)=\left\{\begin{array}{l|l}
v \in \mathcal{C}^{\infty}\left(\mathbb{R} \times[0,1], T^{*} M\right) & \begin{array}{l}
\frac{\partial v}{\partial s}+J_{t}(v) \frac{\partial v}{\partial t}=0 \\
\lim _{s \rightarrow-\infty} v(s, \cdot)=x \\
\lim _{s \rightarrow+\infty} v(s, \cdot)=y
\end{array}
\end{array}\right\}
$$

and

$$
\mathcal{M}^{*}(x, y)= \begin{cases}\mathcal{M}(x, y) & \text { for } x \neq y \\ \mathcal{M}(x, x) \backslash\{x\} & \text { for } x=y .\end{cases}
$$

From [6] and [16], we have

Theorem 3.13.

$$
\mathcal{M}\left(L_{0}, L_{1}\right)=\bigcup_{x, y \in L_{0} \cap L_{1}} \mathcal{M}(x, y) .
$$

Let $\left(\widetilde{J}_{t}\right)$ be a family of almost complex structures on $T^{*} \widetilde{M}$ obtained by lifting the family $\left(J_{t}\right)$ and let $\widetilde{\mathcal{M}}\left(\bar{L}_{0}, \bar{L}_{1}\right), \widetilde{\mathcal{M}}(\widetilde{x}, \widetilde{y})$, and $\widetilde{\mathcal{M}}^{*}(\widetilde{x}, \widetilde{y})$ be the spaces of solutions in $T^{*} \widetilde{M}$ defined in a similar way as in $T^{*} M$. Then these spaces also satisfy Theorem 3.13 and $\widetilde{p}$ maps $\widetilde{\mathcal{M}}\left(\bar{L}_{0}, \bar{L}_{1}\right)$ onto $\mathcal{M}\left(L_{0}, L_{1}\right)$, so that a solution and its image by $\widetilde{p}$ have the same energy.

In the alternative setting, we consider the trajectories of the opposite of the gradient of the one-form $\widehat{\nu}$ with respect to the metric defined on $\Omega(L, L)$ by a family of compatible almost complex structures $\left(\widehat{J}_{t}\right)$.

Denote

$\widehat{\mathcal{M}}(L, L)=\left\{\begin{array}{l|l}v \in \mathcal{C}^{\infty}\left(\mathbb{R} \times[0,1], T^{*} M\right) & \begin{array}{l}\frac{\partial \widehat{v}}{\partial s}+\widehat{J}_{t}(\widehat{v})\left(\frac{\partial \widehat{v}}{\partial t}-X_{t}^{\alpha+d H_{t}(\widehat{v})}\right)=0 \\ v(s, 0) \in L \text { and } v(s, 1) \in L \\ E(v)<\infty\end{array}\end{array}\right\}$,

with the energy defined by the same formula as above.

If $\widehat{v} \in \mathcal{M}(L, L)$, then again

$$
\begin{aligned}
E(\widehat{v}) & =\int_{\mathbb{R} \times[0,1]}\left\|\frac{\partial \widehat{v}}{\partial s}\right\|^{2} d t d s=\int_{\mathbb{R} \times[0,1]} \omega_{M}\left(\frac{\partial \widehat{v}}{\partial s}, \widehat{J} t(\widehat{v}) \frac{\partial \widehat{v}}{\partial s}\right) d t d s \\
& =\int_{\mathbb{R} \times[0,1]} \omega_{M}\left(\frac{\partial \widehat{v}}{\partial s}, \frac{\partial \widehat{v}}{\partial t}-X^{\alpha+d H_{t}}(\widehat{v})\right) d t d s \\
& =\int_{\mathbb{R} \times[0,1]} \omega_{M}\left(\frac{\partial \widehat{v}}{\partial s}, \frac{\partial \widehat{v}}{\partial t}\right) d t d s-\int_{\mathbb{R} \times[0,1]}\left(\alpha+d H_{t}\right)\left(\frac{\partial \widehat{v}}{\partial s}\right) d t d s \\
& =-\int_{\widehat{v}} \widehat{v}
\end{aligned}
$$

and we define analogously the space of solutions $\widehat{\mathcal{M}}(x, y)$ and $\widehat{\mathcal{M}}^{*}(x, y)$. 
Remark 3.14. There is also a correspondence between the two settings for the gradient trajectories. For all $\widehat{v} \in \widehat{\mathcal{M}}(L, L)$, we can associate the map $v$ defined by

$$
v(s, t)=\varphi_{t}^{-1}(\widehat{v}(s, t)) .
$$

Let $\left(J_{t}\right)$ and $\left(\widehat{J}_{t}\right)$ be two families of compatible almost complex structures on $T^{*} M$ such that

$$
\widehat{J_{t}}=\left(\varphi_{t}\right)_{\star} J_{t}\left(\varphi_{t}^{-1}\right)_{\star} .
$$

Then

$$
\frac{\partial v}{\partial s}+J_{t}(v) \frac{\partial v}{\partial t}=\left(\varphi_{t}^{-1}\right)_{\star}\left[\frac{\partial \widehat{v}}{\partial s}+\widehat{J}_{t}(\widehat{v})\left(\frac{\partial \widehat{v}}{\partial t}-X_{t}^{\alpha+d H_{t}(\widehat{v})}\right)\right]
$$

and

$$
E(\widehat{v})=E(v)
$$

so that the map $\widehat{v} \mapsto v$ defines a bijection between $\widehat{\mathcal{M}}(L, L)$ and $\mathcal{M}\left(L_{0}, \varphi_{1}^{-1}\left(L_{0}\right)\right)$.

\subsection{Transversality and compactness}

In order to define our Floer-type homology, let us check now the transversality and compactness requirements.

\subsubsection{Transversality}

We have a classical transversality result of Floer theory:

Theorem 3.15. Assume that $L_{0}$ et $L_{1}$ are transverse.

Then for a generic choice of $J_{t}$, the spaces $\mathcal{M}(x, y)$ are manifolds of finite dimension, of local dimension at $v \in \mathcal{M}(x, y)$ the Maslov-Viterbo index (see 2037) of $v$.

The same result is true for $\widetilde{\mathcal{M}}(\widetilde{x}, \widetilde{y})$ and the map $\widetilde{p}$ induces a diffeomorphism

$$
\widetilde{p}: \widetilde{\mathcal{M}}(\widetilde{x}, \widetilde{y}) \rightarrow \mathcal{M}(x, y)
$$

for $\widetilde{p}(\widetilde{x})=x$ and $\widetilde{p}(\widetilde{y})=y$.

Proof. It is a transversality result analogous to [5, Theorem 3.12] and it can be proved as in [7] (see also [16]).

Remark 3.16. In the case of the alternative setting, a one-form $\alpha+d H_{t}$ being given, there exists a generic Hamiltonian $h_{t}$ (with compact support) such that, if $\left(\psi_{t}\right)$ is the symplectic isotopy spanned by $\alpha+d H_{t}+d h_{t}$, then $L$ and $\psi_{1}(L)$ are transverse.

Using the correspondence 3.14, we are then able to deduce from Theorem 3.15 that the spaces $\widehat{\mathcal{M}}(x, y)$ are submanifolds for a generic choice of family of compatible almost complex structure. 


\subsubsection{Compactness}

Let $x$ and $y$ be two intersection points of $L_{0}$ and $L_{1}$ and let $A>0$. Denote by

$$
\mathcal{M}_{A}^{*}(x, y)=\left\{v \in \mathcal{M}^{*}(x, y) \mid E(v) \leq A\right\}
$$

the space of solutions of finite energy between $x$ and $y$.

The translation in the $s$ variable (defined by $(\sigma \cdot v)(s, t)=v(\sigma+s, t))$ induces a free action of $\mathbb{R}$ on $\mathcal{M}^{*}(x, y)$. Let $\mathcal{L}(x, y)$ denote the quotient $\mathcal{M}^{*}(x, y) / \mathbb{R}$.

Let us recall the result of convergence modulo bubbling of a sequence of elements of $\mathcal{M}_{A}^{*}(x, y)$ (as stated in [16, Proposition 3.7]): if $\left(v_{n}\right)$ is a sequence of elements of $\mathcal{M}_{A}^{*}(x, y)$ with a fixed index equal to $I$, then there exists a subsequence converging (modulo translations, i.e. in the quotient $\mathcal{L}(x, y))$ to a "cusp" curve $\left(\underline{v}, \underline{w}, \underline{u}\right.$ ) (where $\underline{v}$ is a finite collection of solutions $v^{i} \in \mathcal{M}_{A}^{*}\left(z_{i}, z_{i+1}\right), \underline{w}$ is a finite collection of $J$-holomorphic disks $w^{j}$ and $\underline{u}$ is a finite collection of $J$-holomorphic spheres $u^{k}$ ) such that

$$
\begin{aligned}
\sum_{i} \int\left(v^{i}\right)^{\star} \omega+\sum_{j} \int\left(w^{j}\right)^{\star} \omega+\sum_{k} \int\left(u^{k}\right)^{\star} \omega & \leq A \\
\sum_{i} \mu\left(v^{i}\right)+\sum_{j} \mu\left(w^{j}\right)+\sum_{k} 2 c_{1}\left(u^{k}\right) & =\mu_{0} .
\end{aligned}
$$

In our case, the ambient symplectic manifold is the cotangent bundle of the manifold $M$ so that no bubbling of $J$-holomorphic spheres occurs.

Proposition 3.17. Let $L$ be a monotone Lagrangian manifold of Maslov number $N_{L} \geq 3$. Then:

(i) For any sequence of elements $\left(v_{n}\right)$ in the one-dimensional component of $\mathcal{M}_{A}^{*}(x, y)$, there exists a sequence $\left(\sigma_{n}\right)$ of real numbers, such that a subsequence of $\left(\sigma_{n} \cdot v_{n}\right)$ converges in $\mathcal{M}_{A}^{*}(x, y)$;

(ii) if $\left(v_{n}\right)$ is a sequence of elements of $\mathcal{M}_{A}^{*}(x, z)$ of index 2 , then

- either there exists a sequence $\left(\sigma_{n}\right)$ of real numbers such that a subsequence of $\left(\sigma_{n} \cdot v_{n}\right)$ converges to a solution $v$ in $\mathcal{M}_{A}^{*}(x, z)$;

- or there exists a pair of sequences $\left(\left(\sigma_{n}^{1}\right),\left(\sigma_{n}^{2}\right)\right)$ of real numbers and a pair of solutions $\left(v^{1}, v^{2}\right) \in \mathcal{M}_{A}^{*}(x, y) \times \mathcal{M}_{A}^{*}(y, z)$, for some intersection point $y$, such that, for every $i \in\{1 ; 2\}$, a subsequence of $\left(\sigma_{n}^{i} \cdot v_{n}\right)$ converges to $v^{i}$ (in this case one says that $\left(v_{n}\right)$ converges to the broken orbit $\left.\left(v^{1}, v^{2}\right)\right)$.

Remark 3.18. Proposition 3.17 will be used in Section 3.5 to prove the compactness of the space of trajectories. Note that statement (i) in Proposition 3.17 also holds in the case $N_{L}=2$. We will deal with the convergence of a sequence of elements of $\mathcal{M}_{A}^{*}(x, z)$ of index 2 in the case $N_{L}=2$ in the proof of Lemma 3.21.

Proof of Proposition 3.11.

(i) If $\left(v_{n}\right)$ is a sequence of elements of $\mathcal{M}_{A}^{*}(x, y)$ of index 1 , then there exists a subsequence that converges to a ii cusp $i, i$ curve $(\underline{v}, \underline{w}, \emptyset)$ such that

$$
\begin{aligned}
\sum_{i} \int\left(v^{i}\right)^{\star} \omega+\sum_{j} \int\left(w^{j}\right)^{\star} \omega & \leq A \\
\sum_{i} \mu\left(v^{i}\right)+\sum_{j} \mu\left(w^{j}\right) & =1 .
\end{aligned}
$$


Since the area of a $J$-holomorphic disk $w^{j}$ is non-negative, the monotonicity assumption on $L$ (the monotonicity on the disks is sufficient here) gives $\mu\left(w^{j}\right) \geq 3$. Because of (8), there is no bubbling of $J$-holomorphic disk $(\underline{w}=\emptyset)$. Moreover, the dimension of $\mathcal{M}^{*}(x, y)$ is at least 1 (because of the free action of $\mathbb{R}$ ), so that the Maslov class of a solution $v^{i}$ is at least 1 . Hence, the collection $\underline{v}$ can only contain one element that belongs to $v \in \mathcal{M}_{A}^{*}(x, y)$.

(ii) As in (i), no bubbling of $J$-holomorphic disks can occur. As a consequence, there is a subsequence converging to a broken orbit $\underline{v}$ such that

$$
\sum_{i} \mu\left(v^{i}\right)=2
$$

and hence $\underline{v}$ admits at most two components.

For the construction of the complex we need a homotopy lemma:

Lemma 3.19. Let $\left(v_{n}\right)$ be a sequence of elements of $\mathcal{M}_{A}^{*}(x, y)$ of index either 1 or 2 having a subsequence converging either to $\underline{v}=v^{1} \in \mathcal{M}_{A}^{*}(x, y)$ or to $\underline{v}=$ $\left\{v^{1}, v^{2}\right\}$ with $\left(v^{1}, v^{2}\right) \in \mathcal{M}_{A}^{*}(x, y) \times \mathcal{M}_{A}^{*}(y, z)$.

Let $\gamma_{n}:[-\infty ;+\infty] \rightarrow L_{0}$ be the path defined by $\gamma_{n}(s)=v_{n}(s, 0)$ (extended at $s=-\infty$ by $x$ and at $s=+\infty$ by $y$ ). Let $\gamma^{i}:[-\infty ;+\infty] \rightarrow L_{0}$ be the paths defined analogously for the $v^{i}$.

Then, for $n$ large enough, $\gamma_{n}$ is homotopic to either $\gamma^{1}$ (when $\underline{v}=v^{1}$ ) as a path from $x$ to $y$, or to the concatenation of paths $\gamma^{1} \star \gamma^{2}$ (when $\underline{v}=\left\{v^{1}, v^{2}\right\}$ ) as a path from $x$ to $z$.

Proof. The proof is similar to [5, Lemma 3.16] if we add the assumption on the index ensuring that no bubbling of $J$-holomorphic disks can occur.

\subsection{The differential of the Floer complex}

Let $x$ and $y$ be two intersection points of $L_{0}$ and $L_{1}$. In this section, we define an incidence number $[x, y]$.

Let $\mathcal{L}^{0}(x, y)$ be the zero-dimensional component of $\mathcal{L}(x, y)$. For all $z \in$ $L_{0} \cap L_{1}$, fix a lift $\widetilde{z} \in T^{*} \widetilde{M}$. For $g \in \pi_{1}(M)$, denote by $\mathcal{L}_{g}^{0}(x, y) \subset \mathcal{L}^{0}(x, y)$ the subset of trajectories that lift to $\widetilde{\mathcal{L}}\left(\widetilde{x}^{g}, \widetilde{y}\right)$ (with the same notation for the action of $\pi_{1}(M)$ as in Lemma 3.7).

Let us state and prove a lemma that will replace [. Lemma 3.16] in our construction.

Lemma 3.20. Assume that $N_{L} \geq 2$. For all $x, y$ in $L_{0} \cap L_{1}$ and all $g$ in $\pi_{1}(M)$, the set $\mathcal{L}_{g}^{0}(x, y)$ is finite.

If $n_{g}$ denotes the cardinal modulo $\mathbb{Z} / 2$, the number $\sum n_{g} g$ belongs to the Novikov ring $\Lambda_{-u}$.

Proof. The elements of $\mathcal{L}_{g}^{0}(x, y)$ are classes of solutions $v$ which belong to the one-dimensional component of $\mathcal{M}^{*}(x, y)$. We prove that these solutions all have the same energy. For that purpose, we prove that two solutions from $x$ to $y$, which have the same index, and which can be both lifted to trajectories from $\widetilde{x}^{g}$ to $\widetilde{y}$, have the same energy. 
We consider a solution

$$
v:[-\infty,+\infty] \times[0,1] \longrightarrow T^{*} M
$$

in $\mathcal{M}(x, y)$ as a path in $\Omega\left(L_{0}, L_{1}\right)$ from $x$ to $y$. If $v$ is such a path, let

$$
\bar{v}:[-\infty,+\infty] \times[0,1] \longrightarrow T^{*} M
$$

be the "inverse" path defined by

$$
\bar{v}(s, t)=v(-s, t) .
$$

Let $v_{1}$ and $v_{2}$ be two elements of $\mathcal{M}(x, y)$ satisfying $\mu\left(v_{1}\right)=\mu\left(v_{2}\right)$. If we denote by $\gamma=v_{2} \# \bar{v}_{1}$ the concatenation of the paths $v_{2}$ and $\bar{v}_{1}$ (in this order), then $\gamma$ is a loop in $\Omega\left(L_{0}, L_{1}\right)$ based in $x$ (see Figure 3.1). Note that here, we use the notation $\Omega\left(L_{0}, L_{1}\right)$ for the space of paths from $L_{0}$ to $L_{1}$ which are piecewise smooth instead of just smooth as in Section 3.2.

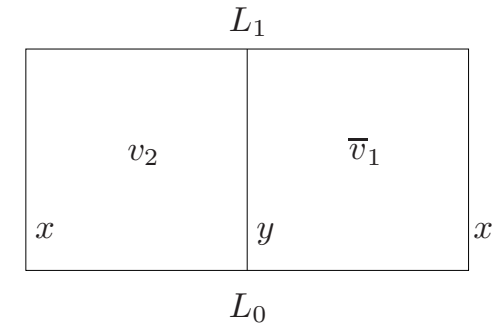

Figure 3.1: the loop $\gamma$

Then

$$
\begin{aligned}
\int_{\gamma} \nu & =-\int \gamma^{\star} \omega_{M} \\
& =-\int v_{2}^{\star} \omega_{M}+\int v_{1}^{\star} \omega_{M} \\
& =E\left(v_{1}\right)-E\left(v_{2}\right) .
\end{aligned}
$$

and thanks to the monotonicity on the loops, by Proposition 3.9,

$$
\int_{\gamma} \nu=k_{L}\left(\mu_{L_{0}}\left(\gamma_{0}\right)-\mu_{L_{1}}\left(\gamma_{1}\right)\right)-u\left(\gamma_{0}\right)
$$

with $\mu_{L_{0}}\left(\gamma_{0}\right)-\mu_{L_{1}}\left(\gamma_{1}\right)=\mu\left(v_{2}\right)-\mu\left(v_{1}\right)=0$.

Moreover, if the lifts of $v_{1}$ and $v_{2}$ are trajectories from $\widetilde{x}^{g}$ to $\widetilde{y}, \gamma_{0}$ can be lifted to a loop based in $\widetilde{x}^{g}$. Therefore, $\gamma_{0}$ is homotopic to the constant loop in $T^{*} M$ and this implies that $u\left(\gamma_{0}\right)=0$ and $\int_{\gamma} \nu=0$. We have thus proved that $v_{1}$ and $v_{2}$ have the same energy.

Assume that $N_{L} \geq 2$. We can then apply Proposition 3.17 and Remark 3.18 a sequence of solutions $\left(v_{n}\right)$ between $x$ and $y$ of Maslov index 1 has a subsequence converging to a solution of $\mathcal{M}^{*}(x, y)$. Thanks to Lemma 3.19, this solution can be lifted to a trajectory from $\widetilde{x}^{g}$ to $\widetilde{y}$. This means that the space $\mathcal{L}_{g}^{0}(x, y)$ is 
a compact space of dimension zero and hence it has only a finite number of elements.

For the second part of the lemma, it is enough to show that for $C>0$, the set

$$
\bigcup_{-u(g) \leq C} \mathcal{L}_{g}^{0}(x, y)
$$

is compact (so that it is finite). A sequence $\left(\underline{v}_{n}\right)$ in this space can be lifted to a sequence $\left(v_{n}\right)$ in the one-dimensional component of $\mathcal{M}^{*}(x, y)$. But if $v_{1}$ and $v_{2}$ are two solutions from $x$ to $y$ with same Maslov class, we have:

$$
E\left(v_{1}\right)-E\left(v_{2}\right)=k_{L}\left(\mu\left(v_{2}\right)-\mu\left(v_{1}\right)\right)-u\left(\gamma_{0}\right)=-u\left(\gamma_{0}\right),
$$

where $\gamma$ denotes the concatenation $v_{2} \# \bar{v}_{1}$ as above.

If $v_{1}$ can be lifted to a trajectory from $\widetilde{x}^{g_{1}}$ to $\widetilde{y}$ and $v_{2}$ to a trajectory from $\widetilde{x}^{g_{2}}$ to $\widetilde{y}, \gamma$ can be lifted as a path from $\widetilde{x}^{g_{2}}$ to $\widetilde{x}^{g_{1}}$ so that $u\left(\gamma_{0}\right)=u\left(g_{2}^{-1} g_{1}\right)$ and

$$
E\left(v_{1}\right)-E\left(v_{2}\right)=u\left(g_{2}\right)-u\left(g_{1}\right) .
$$

As a consequence, if we consider a sequence of solutions $\left(v_{n}\right)$, each $v_{n}$ being lifted as a trajectory from $\widetilde{x}^{g_{n}}$ to $\widetilde{y}$ with $-u\left(g_{n}\right) \leq C$, then:

$$
E\left(v_{n}\right)=E\left(v_{0}\right)-u\left(g_{n}\right)+u\left(g_{0}\right) \leq C+E\left(v_{0}\right)+u\left(g_{0}\right) .
$$

The energy of the elements of this sequence is bounded and we can apply Proposition 3.17: $\left(v_{n}\right)$ has a converging subsequence and the limit of this subsequence can be lifted to a path from $\widetilde{x}^{g_{\infty}}$ to $\widetilde{y}$ which satisfy:

$$
-u\left(g_{\infty}\right)=E\left(v_{\infty}\right)-E\left(v_{0}\right)+u\left(g_{0}\right) \leq C .
$$

This means that $\left(\underline{v_{n}}\right)$ has a converging subsequence in $\bigcup_{-u(g) \leq C} \mathcal{L}_{g}^{0}(x, y)$ which is therefore compact.

We can now define the incidence number:

$$
[x, y]=\sum_{g \in \pi_{1}(M)} n_{g}(x, y) g,
$$

where $n_{g}(x, y)$ is the cardinality of $\mathcal{L}_{g}^{0}(x, y)$. We define the complex $C_{\star}\left(\bar{L}_{0}, \bar{L}_{1}, J_{t}\right)$ as the $\Lambda_{u}$-vector space spanned by the intersection points of $L_{0}$ and $L_{1}$ endowed with the differential:

$$
\partial x=\sum_{g \in \pi_{1}(M), y \in L_{0} \cap L_{1}} n_{g}(x, y) g y .
$$

Lemma 3.21. If $N_{L} \geq 2, \partial \circ \partial=0$.

Proof. In order to prove the relation $\partial^{2}=0$, one has to prove that for all $g \in \pi_{1}(M)$ and all $x, z \in L_{0} \cap L_{1}$, we have:

$$
\sum_{y \in L_{0} \cup L_{1}, g^{\prime}, g^{\prime \prime} \in \pi_{1}(M), g^{\prime} g^{\prime \prime}=g} n_{g^{\prime}}(x, y) n_{g^{\prime \prime}}(y, z)=0 .
$$


When $N_{L} \geq 3$, this is, using Proposition 3.17 as in 5, Lemma 3.18], a consequence of the compactification of the one-dimensional component of $\mathcal{L}_{g}(x, y)$ with broken trajectories (see figure 3.2). This compactification is a compact 1-dimensional manifold whose boundary is

$$
\bigcup_{y \in L_{0} \cup L_{1}, g^{\prime}, g^{\prime \prime} \in \pi_{1}(M), g^{\prime} g^{\prime \prime}=g} \mathcal{L}_{g^{\prime}}^{0}(x, y) \times \mathcal{L}_{g^{\prime \prime}}^{0}(y, z) .
$$

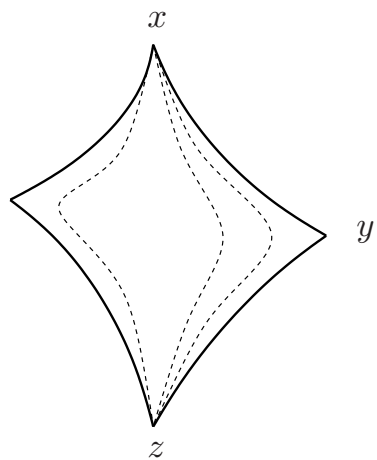

Figure 3.2: The compactification with broken trajectories

Let us consider now the case $N_{L}=2$. Oh noticed in 17 that his extension of the Floer complex to the monotone case is possible under this assumption. This is also possible for the Floer-Novikov complex. Indeed, in the proof that the zero-dimensional component of $\mathcal{L}_{g}(x, y)$ is compact, one only needs that $N_{L} \geq 2$ and it is then possible to define the Floer differential.

The condition $N_{L} \geq 3$ is used to avoid bubbling in the convergence of a sequence of solutions of index 2 and prove that the square of the differential is zero. Let us prove that this is also true for $N_{L}=2$.

The only sequences for which the bubbling of a $J$-holomorphic disk can occur are sequences of solutions from an intersection point $x$ to itself that have Maslov index 2. Then the "bubble" also has Maslov index 2.

In this case, it is possible to compactify the one-dimensional component of $\mathcal{L}_{g}(x, x)$ by adding to the broken trajectories the pairs formed by a constant trajectory and a $J$-holomorphic disk with boundary either on $L_{0}$ or $L_{1}$ (this is similar to 17).

Note also that only a sequence of solutions that can be lifted to paths from $\widetilde{x}$ to $\widetilde{x}$ can converge to a $J$-holomorphic disk, so that this type of compactification is only needed for $\mathcal{L}_{e}(x, x)$, where $e$ is the identity element of $\pi_{1}(M)$. Hence (9) holds also for $g \neq e$ with the previous type of compactification.

When $g=e$, we have as in 17],

$$
\sum_{y \in L_{0} \cup L_{1}, g^{\prime}, g^{\prime \prime} \in \pi_{1}(M), g^{\prime} g^{\prime \prime}=g} n_{g^{\prime}}(x, y) n_{g^{\prime \prime}}(y, z)=\Phi_{L_{0}}(x)+\Phi_{L_{1}}(x)
$$

where $\Phi_{L_{i}}(x)$ is the number (modulo 2) of $J$-holomorphic disks with Maslov index 2 with boundary on $L_{i}$ and that pass through the point $x$. Here we 


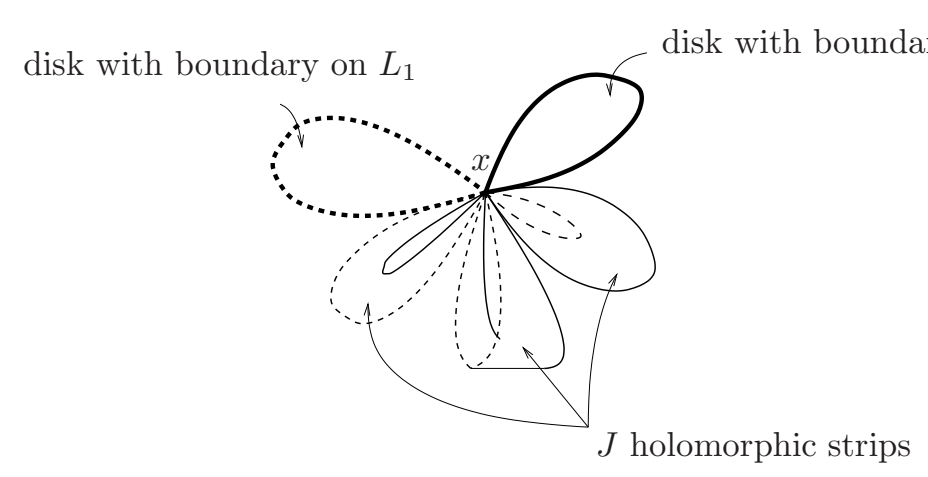

Figure 3.3: The compactification with disks (the plain lines correspond to boundaries on $L_{0}$, the dashed lines to boundaries on $L_{1}$ )

use that $\Phi_{L_{i}}(x)$ is preserved under symplectic isotopies (in [17, Oh uses only Hamiltonian isotopies but the proof is similar with symplectic isotopies) to see that $\Phi_{L_{0}}(x)+\Phi_{L_{i}}(x)=0 \bmod 2$. Therefore, $\partial \circ \partial=0$ even in the case $N_{L}=2$.

Remark 3.22. One can define the same way a complex $C_{\star}\left(\bar{L}, \varphi_{t}, \widehat{J_{t}}\right)$ spanned by the zeros of the one-form $\widehat{\nu}$ and define a differential using the spaces $\widehat{\mathcal{M}}(x, y)$. By the correspondence 3.14, the $\Lambda_{u}$-complexes $C_{\star}\left(\bar{L}, \varphi_{t}, \widehat{J}_{t}\right)$ and $C_{\star}\left(\bar{L}_{0}, \widetilde{\varphi}_{1}{ }^{-1}\left(\bar{L}_{0}\right), J_{t}\right)$ are isomorphic.

\subsection{Hamiltonian invariance}

Denote $H_{\star}\left(\bar{L}_{0}, \bar{L}_{1}, J_{t}\right)$ the homology of the complex $C_{\star}\left(\bar{L}_{0}, \bar{L}_{1}, J_{t}\right)$ defined in Section 3.5. We have assumed that $L_{1}=\varphi_{1}\left(L_{0}\right)$ where the isotopy $\left(\varphi_{t}\right)$ is supposed to be spanned by $\alpha+d H_{t}$ with $\alpha$ a closed one-form on $M$ and $H$ a Hamiltonian with compact support on $T^{*} M \times[0,1]$.

We now prove that this homology does not depend on the generic choice of the pair $\left(J_{t}, H_{t}\right)$. For that purpose, we will use the "alternative" setting and the complex $C_{\star}\left(\bar{L}, \varphi_{t}, J_{t}\right)$. We denote by $H_{\star}\left(\bar{L}, \varphi_{t}^{\alpha+d H_{t}}, J_{t}\right)$ its homology.

Theorem 3.23. For any two generic pairs $\left(H_{t}, J_{t}\right)$ and $\left(H_{t}^{\prime}, J_{t}^{\prime}\right)$, there exists an isomorphism

$$
\Psi: H_{\star}\left(\bar{L}, \varphi_{t}^{\alpha+d H_{t}}, J_{t}\right) \longrightarrow H_{\star}\left(\bar{L}, \varphi_{t}^{\alpha+d H_{t}^{\prime}}, J_{t}^{\prime}\right) .
$$

Proof. As in the proof of [5], define a morphism of $\Lambda_{u}$-complexes

$$
\Psi_{\star}: C_{\star}\left(\bar{L}, \varphi_{t}^{\alpha+d H_{t}}, J_{t}\right) \longrightarrow C_{\star}\left(\bar{L}, \varphi_{t}^{\alpha+d H_{t}^{\prime}}, J_{t}^{\prime}\right)
$$

associated to a family of functions $H_{s, t}: T^{*} M \rightarrow \mathbb{R}$ and a family of compatible almost complex structures $J_{s, t}$ continuous in $(s, t) \in \mathbb{R}^{2}$ and satisfying:

$$
\left(H_{(s, t)}, J_{(s, t)}\right)=\left\{\begin{array}{l}
\left(H_{t}, J_{t}\right) \text { for } s<-R \\
\left(H_{t}^{\prime}, J_{t}^{\prime}\right) \text { for } s>R .
\end{array}\right.
$$


Consider the space

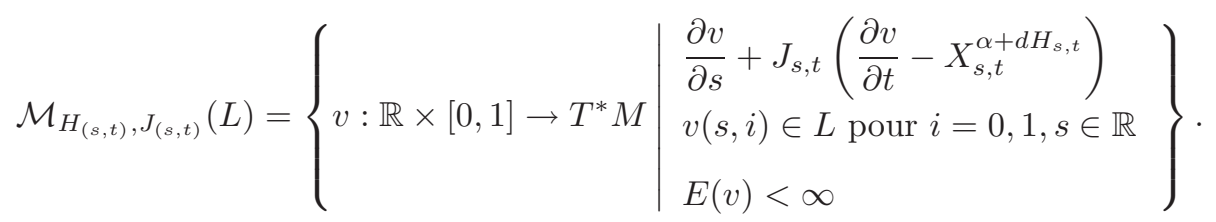

An element $v$ of this space converges to a zero $x$ of the one-form $\widehat{\nu}$ when $s$ goes to $-\infty$ and to a zero $y$ of the one-form $\widehat{\nu}^{\prime}$ (which corresponds to the Hamiltonian $H_{t}^{\prime}$ ) when $s$ goes to $+\infty$. As in Theorem 3.13, we have:

$$
\mathcal{M}_{H_{(s, t)}, J_{(s, t)}}(L)=\bigcup_{x, y} \mathcal{M}_{H_{(s, t)}, J_{(s, t)}}(x, y)
$$

with

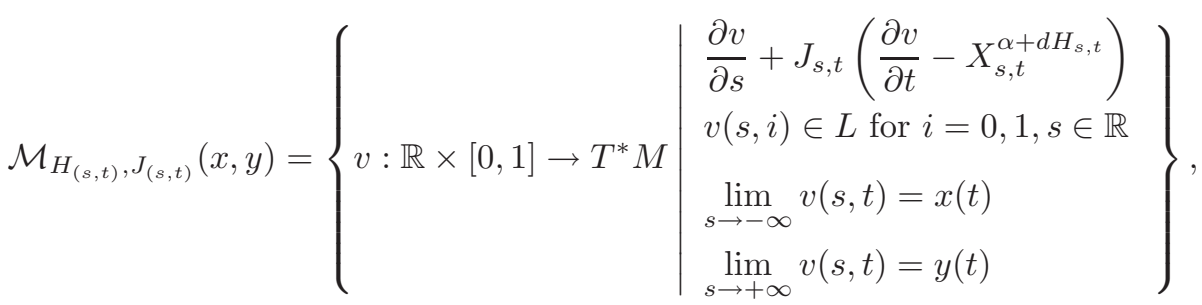

where $x$ (respectively $y$ ) are the zeros of the one-form $\widehat{\nu}$ (respectively $\widehat{\nu}^{\prime}$ ).

We also have a transversality result for these spaces: for a generic choice of the pair $\left(H_{s, t}, J_{s, t}\right)$, the spaces $\mathcal{M}_{H_{(s, t)}, J_{(s, t)}}(x, y)$ are manifolds of local dimension given by the Maslov index of a solution.

We also need a compactness result:

Lemma 3.24. For all $A>0$, the zero-dimensional component of

$$
\mathcal{M}_{H_{(s, t)}, J_{(s, t)}}(x, y ; A)=\left\{v \in \mathcal{M}_{H_{(s, t)}, J_{(s, t)}}(x, y) \mid E(v) \leq A\right\}
$$

is finite.

Proof. The proof is standard since no bubbling occurs in dimension 0 (see e.g. [5. Lemma 3.22]).

As before, we fix a lift $\widetilde{x}$ in $T^{*} \widetilde{M}$ for every zero $x$ of the one-form $\widehat{\nu}$ and a lift $\widetilde{y}$ for every zero $y$ of the one-form $\widehat{\nu}^{\prime}$ (remember that the zeros of $\widehat{\nu}$ are flow trajectories beginning on $L$ and ending on $L)$. Consider for all $g$ of $\pi_{1}(M)$ and all zeros $x$ and $y$ of $\widehat{\nu}$ and $\widehat{\nu}^{\prime}$ respectively, the space $\mathcal{M}_{g, s}(x, y) \subset \mathcal{M}_{H_{(s, t)}, J_{(s, t)}}(x, y)$ of solutions that can be lifted to $T^{*} \widetilde{M}$ in paths from $\widetilde{x}^{g}$ to $\widetilde{y}$. We show that:

Proposition 3.25. For any fixed index I, let

$$
\mathcal{M}_{g, s}^{I}(x, y)=\mathcal{M}_{g, s}(x, y) \cap \mathcal{M}_{H_{(s, t)}, J_{(s, t)}}^{I}(x, y)
$$

be the space of elements of index I in $\mathcal{M}_{g, s}(x, y)$. The energy of any element of $\mathcal{M}_{g, s}^{I}(x, y)$ is bounded from above by some positive constant $A$, that is :

$$
\mathcal{M}_{g, s}^{I}(x, y) \subset \mathcal{M}_{H_{(s, t)}, J_{(s, t)}}(x, y ; A) .
$$


Corollary 3.26. The space $\mathcal{M}_{g, s}^{0}(x, y)$ is finite.

Proof of Proposition 3.2. Here again, we adapt the proof of [5] (see also 12]). We do not have a primitive of $\widehat{\nu}$ but it is possible to bound from above the difference of the energy of two solutions by a constant independent of the solutions.

Consider the norm defined by the compatible metric $\omega_{M}\left(\cdot, J_{(s, t)}\right)$. The energy of a solution $v$ of $\mathcal{M}_{H_{(s, t)}, J_{(s, t)}}(L)$ can be written:

$$
\begin{aligned}
E(v) & =\int_{\mathbb{R} \times[0,1]}\left\|\frac{\partial v}{\partial s}\right\|^{2} d t d s \\
& =\int_{\mathbb{R} \times[0,1]} \omega_{M}\left(\frac{\partial v}{\partial s}, J_{s, t} \frac{\partial v}{\partial s}\right) d t d s \\
& =\int_{\mathbb{R} \times[0,1]} \omega_{M}\left(\frac{\partial v}{\partial s}, \frac{\partial v}{\partial t}-X_{s, t}^{\alpha+d H_{s, t}}\right) d t d s \\
& =\int_{v} \omega_{M}-\int_{\mathbb{R} \times[0,1]}\left(\alpha+d H_{s, t}\right)\left(\frac{\partial v}{\partial s}\right) d t d s .
\end{aligned}
$$

Let $z_{0} \in \Omega(L, L)$ and let $w$ be a fixed path in $\Omega(L, L)$ that joins $y$ to $z_{0}$.

We consider $v:[-\infty,+\infty] \times[0,1]$ as a path in $\Omega(L, L)$ from $x$ to $y$ and we use the concatenation $v \# w$ of $v$ and $w$.

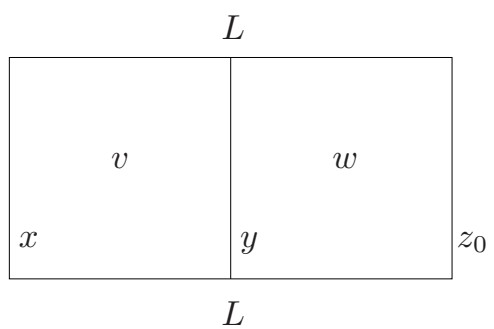

Figure 3.4: The path $v \# w$

Suppose that $w$ has been chosen in such a way that it can be lifted to a path joining $\widetilde{y}$ and $\widetilde{z}_{0}$.

We prove

$$
\int_{v \# w} \widehat{\nu}-\int_{w} \widehat{\nu}^{\prime}=-E(v)+\int_{\mathbb{R} \times[0,1]} \frac{\partial H}{\partial s}(s, t, v) d t d s+C_{1}
$$

where

$$
C_{1}=\int_{[0,1]} H_{t}\left(z_{0}\right)-H_{t}^{\prime}\left(z_{0}\right) d t
$$

is independant of $v$. 
We have:

$$
\begin{aligned}
\int_{v \# w} \widehat{\nu}-\int_{w} \widehat{\nu}^{\prime}= & -\int_{v \# w} \omega_{M}+\int_{\mathbb{R} \times[0,1]}\left(\alpha+d H_{t}\right)\left(\frac{\partial v}{\partial s}\right) d t d s \\
& +\int_{\mathbb{R} \times[0,1]}\left(\alpha+d H_{t}\right)\left(\frac{\partial w}{\partial s}\right) d t d s \\
& +\int_{w} \omega_{M}-\int_{\mathbb{R} \times[0,1]}\left(\alpha+d H_{t}^{\prime}\right)\left(\frac{\partial w}{\partial s}\right) d t d s \\
= & -\int_{v} \omega_{M}+\int_{\mathbb{R} \times[0,1]} \frac{\partial}{\partial s}\left(H_{t}(v)+H_{t}(w)-H_{t}^{\prime}(w)\right) d t d s \\
& +\int_{\mathbb{R} \times[0,1]} \alpha\left(\frac{\partial v}{\partial s}\right),
\end{aligned}
$$

with

$$
\begin{aligned}
\int_{\mathbb{R} \times[0,1]} \frac{\partial}{\partial s} & \left(H_{t}(v)+H_{t}(w)-H_{t}^{\prime}(w)\right) d t d s= \\
& =\int_{[0,1]} H_{t}^{\prime}(y)-H_{t}(x) d t+\int_{[0,1]} H_{t}\left(z_{0}\right)-H_{t}^{\prime}\left(z_{0}\right) d t \\
& =\int_{\mathbb{R} \times[0,1]} \frac{\partial}{\partial s} H_{s, t}(v) d t d s+C_{1} \\
& =\int_{\mathbb{R} \times[0,1]} d H_{s, t}\left(\frac{\partial v}{\partial s}\right) d t d s+\int_{\mathbb{R} \times[0,1]} \frac{\partial H}{\partial s}(s, t, v) d t d s+C_{1} .
\end{aligned}
$$

Consequently

$$
\begin{aligned}
\int_{v \# w} \widehat{\nu}-\int_{w} \widehat{\nu}^{\prime}= & -\int_{v} \omega_{M}+\int_{\mathbb{R} \times[0,1]}\left(\alpha+d H_{s, t}\right)\left(\frac{\partial v}{\partial s}\right) d t d s \\
& +\int_{\mathbb{R} \times[0,1]} \frac{\partial H}{\partial s}(s, t, v) d t d s+C_{1} \\
\int_{v \# w} \widehat{\nu}-\int_{w} \widehat{\nu}^{\prime}= & -E(v)+\int_{\mathbb{R} \times[0,1]} \frac{\partial H}{\partial s}(s, t, v) d t d s+C_{1} .
\end{aligned}
$$

Now, let $v_{1}$ and $v_{2}$ be two elements of $\mathcal{M}_{g, s}(x, y)$. From (10), we have:

$$
\begin{aligned}
E\left(v_{1}\right)-E\left(v_{2}\right)= & -\int_{v_{1} \# w} \widehat{\nu}+\int_{v_{2} \# w} \widehat{\nu} \\
& +\int_{\mathbb{R} \times[0,1]} \frac{\partial H}{\partial s}\left(s, t, v_{1}\right) d t d s-\int_{\mathbb{R} \times[0,1]} \frac{\partial H}{\partial s}\left(s, t, v_{2}\right) d t d s
\end{aligned}
$$

As

$$
\frac{\partial H}{\partial s}: \mathbb{R} \times T^{*} M \rightarrow \mathbb{R}
$$

has compact support, there exists a constant $C_{2} \geq 0$ that does not depend on the $v_{i}(i=1,2)$ such that

$$
\int_{\mathbb{R} \times[0,1]} \frac{\partial H}{\partial s}\left(s, t, v_{1}\right) d t d s-\int_{\mathbb{R} \times[0,1]} \frac{\partial H}{\partial s}\left(s, t, v_{2}\right) d t d s \leq C_{2} .
$$


Moreover, if $\gamma$ is a loop (based in $x$ ) of $\mathcal{M}(L, L)$ obtained by concatenation of the paths $v_{2} \# w$ and $\overline{v_{1} \# w}$, then by the monotonicity condition (and Formula (6)

$$
\begin{aligned}
-\int_{v_{1} \# w} \widehat{\nu}+\int_{v_{2} \# w} \widehat{\nu} & =\int_{\gamma} \widehat{\nu} \\
& =k_{L}\left(\mu_{L}\left(\gamma_{0}\right)-\mu_{L}\left(\gamma_{1}\right)\right)+u\left(\gamma_{0}\right)
\end{aligned}
$$

with $\mu_{L}\left(\gamma_{0}\right)-\mu_{L}\left(\gamma_{1}\right)=\mu\left(v_{2}\right)-\mu\left(v_{1}\right)=0$.

The paths $v_{1}$ and $v_{2}$ belong to $\mathcal{M}_{g, s}(x, y)$, so that $\gamma$ can be lifted to a path from $\widetilde{x}^{g}$ to $\widetilde{x}^{g}$. The path $\gamma_{0}$ is then homotopic to the constant path and we obtain the inequality:

$$
E\left(v_{1}\right)-E\left(v_{2}\right) \leq C_{2} .
$$

This proves that if we fix an element $v_{0}$ in $\mathcal{M}_{g, s}(x, y)$, then for all $v$ in $\mathcal{M}_{g, s}(x, y), E(v) \leq E\left(v_{0}\right)+K$ and consequently $\mathcal{M}_{g, s}(x, y)$ is contained in $\mathcal{M}_{H_{(s, t)}, J_{(s, t)}}(x, y ; A)$ for some positive constant $A$. This proves Proposition 3.25 .

The space $\mathcal{M}_{g, s}^{0}(x, y)$ is thus finite and we can define the morphism of complexes

$$
\Psi_{\star}: C_{\star}\left(\bar{L}, \varphi_{t}^{\alpha+d H_{t}}, J_{t}\right) \longrightarrow C_{\star}\left(\bar{L}, \varphi_{t}^{\alpha+d H_{t}^{\prime}}, J_{t}^{\prime}\right)
$$

by

$$
\Psi_{\star}(x)=\sum_{g \in \pi_{1}(M), y} m_{g}(x, y) g y
$$

where $m_{g}(x, y)$ is the cardinality of $\mathcal{M}_{g, s}^{0}(x, y)$ modulo 2 .

In order to check that the coefficients belong to $\Lambda_{u}$, we use the computations in the proof of 3.25 but this time with $v_{1}$ in $\mathcal{M}_{g_{1}, s}(x, y)$ and $v_{2}$ in $\mathcal{M}_{g_{2}, s}(x, y)$ for two elements $g_{1}$ and $g_{2}$ of $\pi_{1}(M)$. The loop $\gamma$ can then be lifted to a path from $\widetilde{x}^{g_{2}}$ to $\widetilde{x}^{g_{1}}$ so that $u\left(\gamma_{0}\right)=u\left(g_{2}^{-1} g_{1}\right)=-u\left(g_{2}\right)+u\left(g_{1}\right)$ and

$$
E\left(v_{1}\right)-E\left(v_{2}\right) \leq u\left(g_{1}\right)-u\left(g_{2}\right)+C_{2} .
$$

If $v_{0}$ is a fixed element of $\mathcal{M}_{g_{0}, s}(x, y)$, we have for all $v$ of $\mathcal{M}_{g, s}(x, y)$ with $u(g)<C$,

$$
E(v) \leq E\left(v_{0}\right)+C-u\left(g_{0}\right)+C_{2},
$$

and this implies that $\bigcup_{u(g)<C} \mathcal{M}_{g, s}^{0}(x, y)$ is contained in $\mathcal{M}_{H_{(s, t)}, J_{(s, t)}}^{0}(x, y ; A)$ for some positive constant $A$, so that this union is finite.

We use the usual methods of Floer theory to finish the proof of the theorem:

- The fact that $\Psi_{\star}$ commutes with the differentials comes from the study of the compactification with the help of broken trajectories of the 1-dimensionnal component of $\mathcal{M}_{H_{(s, t)}, J_{(s, t)}}(x, y)$.

- The map $\Psi_{\star}$ induces an isomorphism in homology: to prove this, it is enough to consider the morphism defined analogously between

$$
C_{\star}\left(\bar{L}, \varphi_{t}^{\alpha+d H_{t}^{\prime}}, J_{t}^{\prime}\right) \text { and } C_{\star}\left(\bar{L}, \varphi_{t}^{\alpha+d H_{t}}, J_{t}\right)
$$


and to show that the composition of these morphisms are homotopic to the identity.

Notation 3.27. By Theorem 3.23, the homology of the complex $C_{\star}\left(\bar{L}, \varphi_{t}, J_{t}\right)$ only depends on the flux $u$ of the symplectic isotopy $\left(\varphi_{t}\right)$. Hence we will denote its homology by $\mathrm{FH}(\bar{L}, u)$ in the following.

\subsection{Floer-Novikov complex over $\Lambda_{f^{\star} u}$}

Thanks to Lemmata 3.20, 3.21 and 3.19, one can apply [5, Proposition 3.25] and

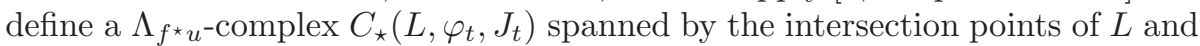
$\varphi_{1}(L)$ such that $f_{\star}: \pi_{1}(L) \rightarrow \pi_{1}(M)$ induces a morphism from $C_{\star}\left(\bar{L}, \varphi_{t}, J_{t}\right)$ to $C_{\star}\left(\bar{L}, \varphi_{t}, J_{t}\right)$ via the ring morphism $f: \Lambda_{f^{\star} u} \rightarrow \Lambda_{u}$.

The differential of this complex is defined for $x$ in $L_{0} \cap L_{1}$ by

$$
\partial x=\sum_{y \in L_{0} \cap L_{1}}[x, y]^{\sim} y
$$

with

$$
[x, y]^{\sim}=\sum_{h \in \pi_{1}(L)} \#_{2} \widetilde{\mathcal{L}}_{h}(x, y) h,
$$

where for any $h \in \pi_{1}(L), \#_{2} \widetilde{\mathcal{L}}_{h}(x, y)$ is the set of paths in $L$ from $x$ to $y$ which lift to the universal covering $\widetilde{L}$ of $L$ to paths from $h \widetilde{x}$ to $\widetilde{y}$. One can prove as in Section 3.6 that the homology of this complex does not depend on the generic choice of the pair $\left(J_{t}, H_{t}\right)$. We will denote this homology $F H(L, u)$.

\section{Floer homology and Novikov theory}

In this section, we prove Theorem 1.4 of the introduction. We deduce this theorem from an analogous result which relates $F H(\bar{L}, u)$ and the Novikov homology $H\left(\bar{L}, f^{\star} u\right)$ of $L$ associated to $f^{\star} u$ and the covering $\bar{L} \rightarrow L$ (defined in Lemma 3.7 as the pull-back of the covering $T^{*} \widetilde{M} \rightarrow T^{*} M$, see also Remark 2.4).

\subsection{Relation between $F H(\bar{L}, u)$ and $H\left(\bar{L}, f^{\star} u\right)$}

We first prove that the Floer-Novikov homology $F H(\bar{L}, u)$ is invariant by small rescaling of $u$ :

Proposition 4.1. Let $u$ be an element of $H^{1}(M)$.

Assume that the Lagrangian submanifold $L$ is monotone on the loops with Maslov number $N_{L} \geq 2$.

Then there exists $\varepsilon>0$ such that for every real number $\sigma$ satisfying $|\sigma|<\varepsilon$,

$$
F H(\bar{L},(1+\sigma) u) \simeq F H(\bar{L}, u) .
$$

Proof. In the exact case, Damian uses the symplectic isotopy of $T^{*} L$ spanned by a 1 -form in the class of $f^{\star} u$. Thanks to the exactness of $L$, he extends it to $T^{*} M$, so that the zeros of the associated one-form are constant paths. This is particularly useful for the choice of a one-form representing $u$ in the definition of $F H(\bar{L},(1+\sigma) u)$. 
In the monotone case, we cannot use this result on the extension of symplectic isotopies of $T^{*} L$ to $T^{*} M$. But, what we actually need for the proof (see [5] and also [12]) is a symplectic isotopy with the property that the projection of the flow trajectories on the base space $M$ (and in particular the projection of the zeros of the one-form $\widehat{\nu}$ associated to this isotopy) lie in "small" balls.

More precisely, let $\alpha$ be a closed one-form in the class of $u$ and $J$ be a fixed compatible almost complex structure. Assume that this almost complex structure induces a complete metric $g_{J}$ on $T^{*} M$.

Lemma 4.2. There exists a constant $c_{1}>0$ such that, for every $\delta>0$, there exists a Hamiltonian

$$
H:[0,1] \times T^{*} M \rightarrow \mathbb{R}
$$

with compact support and a family of almost complex structures $\left(J_{t}\right)$ satisfying $\left\|H_{t}\right\|_{\epsilon}<\delta$ and $\left\|J_{t}-J\right\|<\delta$ such that:

(i) the pair $\left(\alpha+d H_{t}, J_{t}\right)$ satisfies the transversality assumption;

(ii) the canonical projection $\pi$ of $T^{*} M$ maps a zero $x$ of the one-form $\widehat{\nu}$ associated to the symplectic isotopy spanned by $\alpha+d H_{t}$ into a ball in $M$ centered in $\pi(x(0))$ with radius $c_{1} \delta$.

The norm \|\|$_{\epsilon}$ is the usual norm on the Hamiltonians used in transversality results (see [7], [12]):

$$
\|h\|_{\epsilon}=\sum_{k=0}^{\infty} \epsilon_{k}\|h\|_{C^{k}\left([0,1] \times T^{*} M\right)}
$$

where $\epsilon_{k}>0$ is a sufficiently rapidly decreasing sequence.

Proof. The symplectic isotopy $\varphi_{t}^{\alpha}$ of $T^{*} M$ spanned by the symplectic dual $X^{\alpha}$ of $\alpha$ can be written:

$$
\varphi_{t}^{\alpha}(p, q)=\left(p+t \alpha_{q}, q\right)
$$

Note that the trajectory $\varphi_{t}^{\alpha}\left(p_{0}, q_{0}\right)$ of $X^{\alpha}$ in $T^{*} M$ with initial condition the point $\left(p_{0}, q_{0}\right)$ lies in the fiber of $q_{0} \in M$.

Note also that $\varphi_{t}^{\alpha}$ does not necessarily satisfy the transversality assumption between $L$ and $\varphi_{1}^{\alpha}(L)$ so that it may not be possible to use this isotopy for the description of $H F(L, u)$.

Nevertheless, by the transversality theorem (Theorem 3.15) and Remark 3.16, for every $\delta>0$, there exists a Hamiltonian $H:[0,1] \times T^{*} M \rightarrow \mathbb{R}$ with compact support and a family of almost complex structures $\left(J_{t}\right)$ satisfying $\left\|H_{t}\right\|_{\epsilon}<\delta$ and $\left\|J_{t}-J\right\|<\delta$ and such that the pair $\left(\alpha+d H_{t}, J_{t}\right)$ satisfies the transversality assumption.

Let $\zeta(t)$ be the trajectory of $X^{\alpha+d H_{t}}$ with initial condition the point $\left(p_{0}, q_{0}\right)$ of $T^{*} M$. Denote by $q(t)$ its image in $M$ by the canonical projection $\pi: T^{*} M \rightarrow$ $M$ of the cotangent bundle. We have:

$$
\frac{d}{d t} q(t)=T_{\zeta(t)} \pi\left(\frac{d}{d t} \zeta(t)\right)=T_{\zeta(t)} \pi\left(X^{\alpha}(\zeta(t))+X^{d H_{t}}(\zeta(t))\right) .
$$

Since the isotopy $\varphi_{t}^{\alpha}$ spanned by $X^{\alpha}$ satisfies: $\pi\left(\varphi_{t}^{\alpha}\right)(p, q)=q$,

$$
T_{\zeta(t)} \pi\left(X^{\alpha}(\zeta(t))\right)=0
$$


so that:

$$
\frac{d}{d t} q_{t}=T_{\zeta(t)} \pi\left(X^{d H_{t}}(\zeta(t))\right) .
$$

Moreover, for all $z \in T^{*} M$, we have:

$$
\begin{aligned}
\left\|X^{d H_{t}}(z)\right\|^{2} & =d_{z} H_{t}\left(-J(z) X^{d H_{t}}(z)\right) \\
& \leq\left\|d_{z} H_{t}\right\|\left\|-J(z) X^{d H_{t}}(z)\right\|=\left\|d_{z} H_{t}\right\|\left\|X^{d H_{t}}(z)\right\|
\end{aligned}
$$

for the norm associated to the scalar product $g_{J}$. Hence,

$$
\left\|X^{d H_{t}}(z)\right\| \leq\left\|d_{z} H_{t}\right\| \leq \frac{\delta}{\epsilon_{1}}
$$

(where $\epsilon_{1}$ is the first term of the sequence defining the norm \|\|$_{\epsilon}$ ).

Notice that $\left\|T_{\zeta(t)} \pi\right\|$ is bounded on $T^{*} M$ : it is bounded on each trivialising open set for the cotangent bundle $T^{*} M \rightarrow M$; the base space $M$ being compact, it is bounded on the whole $T^{*} M$. Thus, there exists a constant $c_{1}$ (which does not depend on $H$ ) such that,

$$
\left\|\frac{d}{d t} q_{t}\right\| \leq\left\|T_{\zeta(t)} \pi\right\|\left\|X^{d H_{t}}(\zeta(t))\right\| \leq c_{1} \delta,
$$

and consequently, for all $\tau \in[0,1]$, we have:

$$
d\left(q(\tau), q_{0}\right) \leq \int_{0}^{\tau}\left\|\frac{d}{d t} q_{t}\right\| \leq \tau c_{1} \delta .
$$

This means that, on $[0,1]$, the trajectories of $X^{\alpha+d H_{t}}$ lie in the fibers of the points of $M$ which belongs to the ball of radius $c_{1} \delta$ centered in the projection of the initial condition (see figure 4.1).

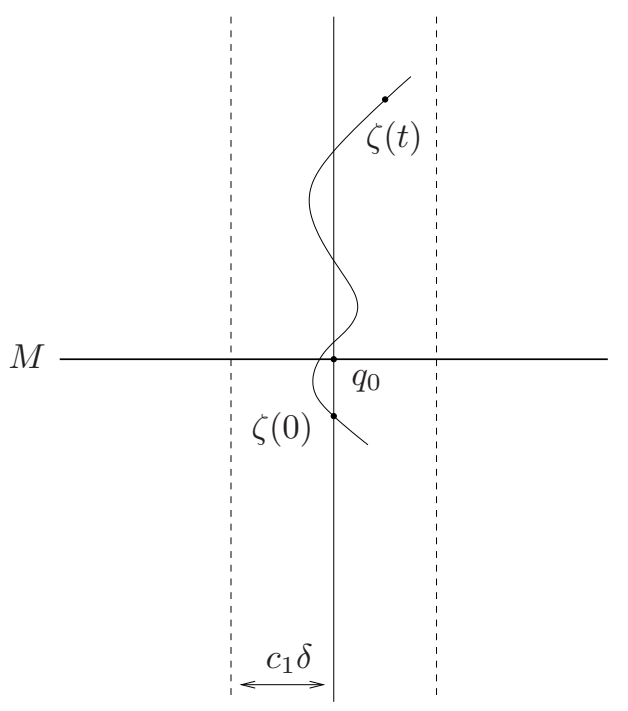

Figure 4.1: the trajectory $\zeta$

This is in particular true for the zeros $x_{i}$ of the one-form $\widehat{\nu}$. 
Let $\mathcal{V}_{i}$ be a neighbourhood of each trajectory $x_{i}$ such that the projection of $\mathcal{V}_{i}$ is contained in $B\left(\pi\left(x_{i}(0)\right), c_{1} \delta\right)$. Denote $\mathcal{V}$ the union of the $\mathcal{V}_{i}$.

Lemma 4.3. There exists $c_{2}>0$ such that for all $z \in \Omega(L, L)$ whose image is not contained in $\mathcal{V}$,

$$
\left\|z^{\prime}(t)-X^{\alpha+d H_{t}}(z(t))\right\|_{L^{2}} \geq c_{2}
$$

Proof. The proof is analogous to [5, Lemma 4.4]. Assume the contrary: there exists a sequence $\left(z_{n}\right) \in \Omega(L, L)$ of paths whose images are not contained in $\mathcal{V}$ such that

$$
\lim _{n \rightarrow+\infty}\left\|z_{n}^{\prime}(t)-X^{\alpha+d H_{t}}\left(z_{n}(t)\right)\right\|_{L^{2}}=0
$$

One has then to prove that this sequence admits a subsequence converging to a zero of $\widehat{\nu}$. This contradicts the fact that the images of the $z_{n}$ 's are not contained in $\mathcal{V}$.

We can now choose $\delta>0$ small enough so that there exists a closed one-form $\eta \in u$ such that

$$
\eta=0 \text { on } \bigcup_{i} B\left(\pi\left(x_{i}(0)\right), c_{1} \delta\right)
$$

We also fix $\varepsilon>0$ such that $\varepsilon\|\eta\|<c_{2} / 3$. In particular, the one-form (still denoted $\eta$ ) lifted to $T^{*} M$ is zero on $\mathcal{V}$, a property that we need in the computations (see Proposition 4.4, and also [5], [12]).

Choose a real number $\sigma<\varepsilon$ and consider the isotopy $\varphi_{t}^{\alpha+\sigma \eta+d H_{t}}$ spanned by $X^{\alpha+\sigma \eta+d H_{t}}$. The constant $\varepsilon$ is chosen small enough so that $\varphi_{1}^{\alpha+\sigma \eta+d H_{t}}(L)$ is transverse to $L$.

Applying the transversality theorem 3.15 to $\alpha+\sigma \eta+d H_{t}$, we deduce the existence of a compatible almost complex structure $J_{t}^{\prime}$ such that $\left\|J_{t}^{\prime}-J\right\|<\delta$ and such that the pair $\left(\alpha+\sigma \eta+d H_{t}, J_{t}^{\prime}\right)$ satisfies the transversality assumption.

Since $\Lambda_{u}=\Lambda_{\tau u}$ for all $\tau>0$, we can define the $\Lambda_{u}$-complexes

$$
C_{\star}\left(\bar{L}, \varphi_{t}^{\alpha+d H_{t}}, J_{t}\right) \text { and } C_{\star}\left(\bar{L}, \varphi_{t}^{\alpha+\sigma \eta+d H_{t}}, J_{t}^{\prime}\right) .
$$

Let us prove that the homologies of this complexes are isomorphic. This will achieve the proof of Proposition 4.1.

As in Section 3.6, we define a morphism of complexes associated to a homotopy between the pairs $\left(\alpha+d H_{t}, J_{t}\right)$ and $\left(\alpha+d H_{t}+\sigma \eta, J_{t}^{\prime}\right)$. Let $\chi$ be a monotone increasing function on $\mathbb{R}$ that vanishes for $s \leq-R$ and is equal to 1 for $s \geq R$. Let $J_{s, t}$ be a homotopy of compatible almost complex structures such that $J_{s, t}=J_{t}$ for $s \leq-R, J_{s, t}=J_{t}^{\prime}$ for $s \geq R$ and $\left\|J_{s, t}-J\right\|<\delta$. The homotopy is defined as:

$$
\left(\alpha+\chi(s) \sigma \eta+d H_{t}, J_{t, s}\right) .
$$

Consider the space of solutions

$$
v: \mathbb{R} \times[0,1] \longrightarrow T^{*} M
$$


of finite energy $E(v)$ (for the norm defined defined by $g_{J}$ ) of the partial differential equation:

$$
\frac{\partial v}{\partial s}+J_{s, t}\left(\frac{\partial v}{\partial t}-X_{s, t}^{\alpha+\chi(s) \sigma \eta+d H_{s, t}}(v)\right)=0
$$

such that $v(s, i) \in L$ for $i=0,1$.

These solutions converge to an orbit of $X^{\alpha+d H_{t}}$ (respectively of $X^{\alpha+\sigma \eta+d H_{t}}$ ) when $s$ goes to $-\infty$ (respectively $+\infty$ ).

We define as in the previous sections the spaces $\mathcal{M}_{\chi, J_{(s, t)}}(x, y)$ of solutions between two orbits $x$ and $y$. By transversality, these spaces are manifolds the local dimension of which is given by the Maslov class.

Let $\mathcal{M}_{g, s}(x, y) \subset \mathcal{M}_{\chi, J_{s, t}}(x, y)$ be the space of solutions that can be lifted to $T^{*} \widetilde{M}$ in paths from $\widetilde{x}^{g}$ to $\widetilde{y}$ (for fixed lifts $\widetilde{x}$ and $\widetilde{y}$ ). In the following, we prove that the zero-dimensional component of $\mathcal{M}_{g, s}(x, y)$ is compact, so that we can define a morphism

$$
\Gamma_{\star}: C_{\star}\left(\bar{L}, \varphi_{t}^{\alpha+d H_{t}}, J_{t}\right) \longrightarrow C_{\star}\left(\bar{L}, \varphi_{t}^{\alpha+\sigma \eta+d H_{t}}, J_{t}^{\prime}\right)
$$

by

$$
\Gamma_{\star}(x)=\sum_{g \in \pi_{1}(M), y} m_{g}(x, y) g y
$$

where $m_{g}(x, y)$ is the cardinality (modulo 2 ) of the space $\mathcal{M}_{g, s}^{0}(x, y)$.

We prove now the compactness of $\mathcal{M}_{g, s}^{0}(x, y)$ and we check that

$$
\sum_{g \in \pi_{1}(M), y} m_{g}(x, y) g \in \Lambda_{u}
$$

Proposition 4.4. For any fixed index $I$, the energy of any element of $\mathcal{M}_{g, s}^{I}(x, y)$ is bounded from above by some positive constant $A$.

Proof. Let $v_{1}$ and $v_{2}$ be two elements of $\mathcal{M}_{g, s}(x, y)$ with the same Maslov index $I$. If $\gamma: \mathbb{R} \times[0,1] \rightarrow T^{*} M$ is the concatenation of $v_{2}$ and $\overline{v_{1}}$, then $\gamma$ is, after reparametrization in the $s$ variable, a loop $\Omega(L, L)$ based at $x$.

We have:

$$
\begin{aligned}
\int \widehat{\nu}\left(\frac{\partial \gamma}{\partial s}\right) d s= & -\int_{-\infty}^{+\infty} \int_{0}^{1}\left\langle\frac{\partial v_{1}}{\partial s}, J\left(\frac{\partial v_{1}}{\partial t}-X^{\alpha+d H_{t}}\left(v_{1}\right)\right)\right\rangle d t d s \\
& +\int_{-\infty}^{+\infty} \int_{0}^{1}\left\langle\frac{\partial v_{2}}{\partial s}, J\left(\frac{\partial v_{2}}{\partial t}-X^{\alpha+d H_{t}}\left(v_{2}\right)\right)\right\rangle d t d s
\end{aligned}
$$

We prove that:

1) for any $s \in \mathbb{R}$,

$$
-\int_{0}^{1}\left\langle\frac{\partial v_{1}}{\partial s}, J\left(\frac{\partial v_{1}}{\partial t}-X^{\alpha+d H_{t}}\left(v_{1}\right)\right)\right\rangle d t \geq \frac{1}{3}\left\|\frac{\partial v}{\partial s}\right\|_{L^{2}}^{2}
$$

2) for any $s \in \mathbb{R}$,

$$
-\int_{0}^{1}\left\langle\frac{\partial v_{2}}{\partial s}, J\left(\frac{\partial v_{2}}{\partial t}-X^{\alpha+d H_{t}}\left(v_{2}\right)\right)\right\rangle d t \leq \frac{5}{3}\left\|\frac{\partial v_{2}}{\partial s}\right\|_{L^{2}}^{2} .
$$


Using 1) and 2), we will deduce that

$$
\frac{1}{3} E\left(v_{1}\right) \leq u\left(\gamma_{0}\right)+\frac{5}{3} E\left(v_{2}\right)
$$

and use this inequality to achieve the proof of Proposition 4.4.

Proof of 1): If $v$ is an element of $\mathcal{M}_{g, s}(x, y)$, we have:

$$
\begin{aligned}
\int_{0}^{1}\left\langle\frac{\partial v}{\partial s}, J\right. & \left.\left(\frac{\partial v}{\partial t}-X^{\alpha+d H_{t}}(v)\right)\right\rangle d t= \\
= & \int_{0}^{1}\left\langle\frac{\partial v}{\partial s}, J\left(J_{s, t} \frac{\partial v}{\partial s}+X^{\alpha+\chi(s) \sigma \eta+d H_{t}}(v)-X^{\alpha+d H_{t}}(v)\right)\right\rangle d t \\
= & \int_{0}^{1}\left\langle\frac{\partial v}{\partial s}, J J_{s, t} \frac{\partial v}{\partial s}\right\rangle d t+\int_{0}^{1}\left\langle\frac{\partial v}{\partial s}, J X^{\chi(s) \sigma \eta}(v)\right\rangle d t \\
= & -\int_{0}^{1}\left\langle J \frac{\partial v}{\partial s}, J_{s, t} \frac{\partial v}{\partial s}\right\rangle d t-\int_{0}^{1} \omega_{M}\left(\frac{\partial v}{\partial s}, X^{\chi(s) \sigma \eta}(v)\right) d t \\
= & -\int_{0}^{1}\left\|\frac{\partial v}{\partial s}\right\|^{2} d t-\int_{0}^{1}\left\langle J \frac{\partial v}{\partial s},\left(J_{s, t}-J\right) \frac{\partial v}{\partial s}\right\rangle d t \\
& -\int_{0}^{1} \chi(s) \sigma \eta\left(\frac{\partial v}{\partial s}\right) d t
\end{aligned}
$$

- Assume firstly that $v(s, \cdot)$ takes values in $\mathcal{V}$. Since $\eta$ vanishes on this neighbourhood of the zeros, we have:

$$
\begin{aligned}
\int_{0}^{1}\left\langle\frac{\partial v}{\partial s},\right. & \left.J\left(\frac{\partial v}{\partial t}-X^{\alpha+d H_{t}}(v)\right)\right\rangle d t= \\
& =-\int_{0}^{1}\left\|\frac{\partial v}{\partial s}\right\|^{2} d t+\int_{0}^{1}\left\langle J \frac{\partial v}{\partial s},\left(J-J_{s, t}\right) \frac{\partial v}{\partial s}\right\rangle d t \\
& \leq-\int_{0}^{1}\left\|\frac{\partial v}{\partial s}\right\|^{2} d t+\delta \int_{0}^{1}\left\|\frac{\partial v}{\partial s}\right\|^{2} d t \\
& \leq-(1-\delta) \int_{0}^{1}\left\|\frac{\partial v}{\partial s}\right\|^{2} d t
\end{aligned}
$$

We can assume that $\delta<2 / 3$, so that:

$$
\int_{0}^{1}\left\langle\frac{\partial v}{\partial s}, J\left(\frac{\partial v}{\partial t}-X^{\alpha+d H_{t}}(v)\right)\right\rangle d t \leq-\frac{1}{3} \int_{0}^{1}\left\|\frac{\partial v}{\partial s}\right\|^{2} d t .
$$

- If $v(s, \cdot)$ does not take its values in $\mathcal{V}$, by the same argument as in [5] and using Lemma 4.3, we also have in this case that:

$$
\int_{0}^{1}\left\langle\frac{\partial v}{\partial s}, J\left(\frac{\partial v}{\partial t}-X^{\alpha+d H_{t}}(v)\right)\right\rangle d t \leq-\frac{1}{3}\left\|\frac{\partial v}{\partial s}\right\|_{L^{2}}^{2},
$$

if we choose $\delta$ small enough. 
Applying this to $v_{1}$ we get 13 and integrating in the $s$ variable:

$$
\frac{1}{3} E\left(v_{1}\right) \leq-\int_{-\infty}^{+\infty} \int_{0}^{1}\left\langle\frac{\partial v_{1}}{\partial s}, J\left(\frac{\partial v_{1}}{\partial t}-X^{\alpha+d H_{t}}\left(v_{1}\right)\right)\right\rangle d t d s .
$$

Proof of 2): We use the relation (16):

$$
\begin{aligned}
-\int_{0}^{1}\left\langle\frac{\partial v_{2}}{\partial s}\right. & \left., J\left(\frac{\partial v_{2}}{\partial t}-X^{\alpha+d H_{t}}\left(v_{2}\right)\right)\right\rangle d t= \\
= & \int_{0}^{1}\left\|\frac{\partial v_{2}}{\partial s}\right\|^{2} d t+\int_{0}^{1}\left\langle J \frac{\partial v_{2}}{\partial s},\left(J-J_{s, t}\right) \frac{\partial v_{2}}{\partial s}\right\rangle d t \\
& +\int_{0}^{1} \chi(s) \sigma(-\eta)\left(\frac{\partial v_{2}}{\partial s}\right) d t .
\end{aligned}
$$

We distinguish again the two cases:

- either $v_{2}(s, \cdot)$ takes its values in $\mathcal{V}$, so that we have:

$$
-\int_{0}^{1}\left\langle\frac{\partial v_{2}}{\partial s}, J\left(\frac{\partial v_{2}}{\partial t}-X^{\alpha+d H_{t}}\left(v_{2}\right)\right)\right\rangle d t \leq(1+\delta)\left\|\frac{\partial v_{2}}{\partial s}\right\|_{L^{2}}^{2},
$$

- or $v_{2}(s, \cdot)$ does not take all its values in $\mathcal{V}$ and we use Lemma 4.3. Firstly, we have:

$$
-\int_{0}^{1}\left\langle\frac{\partial v_{2}}{\partial s}, J\left(\frac{\partial v_{2}}{\partial t}-X^{\alpha+d H_{t}}\left(v_{2}\right)\right)\right\rangle d t \leq(1+\delta)\left\|\frac{\partial v_{2}}{\partial s}\right\|_{L^{2}}^{2}+\frac{c_{2}}{3}\left\|\frac{\partial v_{2}}{\partial s}\right\|_{L^{2}} .
$$

By a consequence of Lemma 1.3 (see [5):

$$
\frac{c_{2}}{3}\left\|\frac{\partial v_{2}}{\partial s}\right\|_{L^{2}} \leq\left(\frac{2}{3}-\delta\right)\left\|\frac{\partial v_{2}}{\partial s}\right\|_{L^{2}}^{2} .
$$

In both cases, we have (14) and by integration:

$$
-\int_{-\infty}^{+\infty} \int_{0}^{1}\left\langle\frac{\partial v_{2}}{\partial s}, J\left(\frac{\partial v_{2}}{\partial t}-X^{\alpha+d H_{t}}\left(v_{2}\right)\right)\right\rangle d t d s \leq \frac{5}{3} E\left(v_{2}\right) .
$$

To prove (15), we use that

$$
\int_{-\infty}^{+\infty} \widehat{\nu}\left(\frac{\partial \gamma}{\partial \sigma}\right)
$$

is the value of the one-form $\widehat{\nu}$ on the loop $\gamma$ based in $x$. Thanks to (6), this term is equal to $u\left(\gamma_{0}\right)$ if the two solutions have the same Maslov class.

By assumption, $v_{1}$ and $v_{2}$ can be lifted to paths between $\widetilde{x}^{g}$ and $\widetilde{y}$, the loop $\gamma_{0}$ is homotopic to the constant loop based in $x$.

We thus have, fixing an element $v_{0}$ in $\mathcal{M}_{g, s}^{I}(x, y)$, for all $v$ of $\mathcal{M}_{g, s}^{I}(x, y)$,

$$
E(v) \leq 5 E\left(v_{0}\right) .
$$

To end the proof of Proposition 4.4, we choose $A=5 E\left(v_{0}\right)$. 
Looking at the proof of Proposition 4.4, we see that the sum

$$
\sum_{g \in \pi_{1}(M)} m_{g}(x, y) g \in \Lambda_{u} .
$$

Indeed, if $v_{1}$ can be now lifted as a path from $\widetilde{x}^{g_{1}}$ to $\widetilde{y}$ and $v_{2}$ as a path from $\widetilde{x}^{g_{2}}$ to $\widetilde{y}$, we have $u\left(\gamma_{0}\right)=u\left(g_{1}\right)-u\left(g_{2}\right)$, so that (15) becomes:

$$
\frac{1}{3} E\left(v_{1}\right) \leq u\left(g_{1}\right)-u\left(g_{2}\right)+\frac{5}{3} E\left(v_{2}\right) .
$$

This implies that if $v_{0}$ is a fixed element of $\mathcal{M}_{g_{0}, s}(x, y)$, we have then for all $v$ of $\mathcal{M}_{g, s}(x, y)$ with $u(g)<C$,

$$
E(v) \leq 3\left(C-u\left(g_{0}\right)\right)+5 E\left(v_{0}\right)
$$

and this prove that

$$
\bigcup_{u(g)<C} \mathcal{M}_{g, s}^{0}(x, y) \subset \mathcal{M}_{\chi, J_{s, t}}(x, y ; A)
$$

for the positive constant $\left.A=3\left(C-u\left(g_{0}\right)\right)+5 E\left(v_{0}\right)\right)$.

The map $\Gamma_{\star}$ is a morphism of complexes. This is a consequence of the compactification of the one-dimensional component of $\mathcal{M}_{g, s}(x, y)$ by broken trajectories $\left(v^{1}, v^{2}\right)$, where

- one of the $v^{i}$ s satisfies (12);

- the other is solution of the Floer equation corresponding to $\left(\alpha+d H_{t}, J_{t}\right)$ or $\left(\alpha+d H_{t}+\sigma \eta, J_{t}^{\prime}\right)$.

As in Theorem 3.23, we use the usual methods of Floer theory to prove that the morphism of complexes $\Gamma_{\star}$ that induces an isomorphism in homology.

Now, we prove that, with an additional assumption on the Maslov number of $L$, for $\sigma$ small enough, $F H(\bar{L}, \sigma u)$ is the Novikov homology of $L$ associated to $f^{\star} u$ and the covering $\bar{L} \rightarrow L$.

Proposition 4.5. Let $u$ be an element of $H^{1}(M)$.

Assume that the Lagrangian submanifold $L$ is monotone on the loops and that its Maslov number $N_{L}$ satisfies

$$
N_{L} \geq \operatorname{dim}(M)+2 .
$$

Then there exists $\varepsilon>0$ (depending on u) such that for all real number $\sigma$ satisfying $|\sigma|<\varepsilon$,

$$
F H(\bar{L}, \sigma u) \simeq H\left(\bar{L}, f^{\star} u\right) .
$$

Proof. We begin (as in 河) by substituting in the proof of Proposition 4.1 the one-form $\alpha \in u$ by an exact one-form $d g$ where $g: M \rightarrow \mathbb{R}$. For $\sigma$ small enough, we obtain a morphism of $\Lambda_{u}$-complexes:

$$
\Gamma_{\star}^{0}: C_{\star}\left(\bar{L}, \varphi_{t}^{d g+d H_{t}}, J_{t}\right) \longrightarrow C_{\star}\left(\bar{L}, \varphi_{t}^{d g+\sigma \eta+d H_{t}}, J_{t}^{\prime}\right)
$$


which induces an isomorphism in homology. The first complex is a $\Lambda$-complex whose coefficients have been extended to $\Lambda_{u}$. There is a natural isomorphism

$$
C_{\star}\left(\bar{L}, \varphi_{t}^{d g+d H_{t}}, J_{t}\right) \simeq \Lambda_{u} \otimes_{\Lambda} C_{\star}\left(\bar{L}, \varphi_{t}^{d g+d H_{t}}, J_{t}\right) .
$$

By Hamiltonian invariance, we know that the homotopy type of the complex $C_{\star}\left(\bar{L}, \varphi_{t}^{d g+d H_{t}}, J_{t}\right)$ does not depend on a regular choice of the pair $\left(H_{t}^{0}, J_{t}^{0}\right)$.

In order to define an isomorphism between $F H(\bar{L}, \sigma u)$ and $H_{\star}(\bar{L}, u)$, we use an other choice of Hamiltonian. For that purpose, in the monotone case, we need to adapt a construction of Oh (18). We consider a local Floer homology, namely the Floer homology in a Darboux neighbourhood $\mathcal{U}$ of $L$ in $T^{*} M$. Considering $\mathcal{U}$ as the neighbourhood of the zero section in $T^{*} L$, we can define the Hamiltonian $\mathcal{H}=h \circ \pi_{L}$ on $\mathcal{U}$, where $h$ is a Morse function on $L$ and $\pi_{L}: T^{*} L \rightarrow L$ is the canonical projection of the cotangent.

If $h$ is small enough (in the $C^{2}$-topology) and if its gradient for a metric on $L$ is Morse-Smale, the local Floer complex is spanned by the intersection points of $L$ and its displacement $L+d h$ and we have a bijection between the $J$-holomorphic strips of the Floer homology and the trajectories of the gradient of $h$ which define the Morse differential.

To go back to $T^{*} M$, it is then enough to extend the Hamiltonian $\mathcal{H}$ to $T^{*} M$ (setting $\mathcal{H}=0$ outside a neighbourhood containing $\mathcal{U}$ ). Oh has proved that under the assumption that $L$ is monotone (on the disks) in $T^{*} M$ and $N_{L} \geq$ $\operatorname{dim}(M)+2$, a Floer trajectory in $T^{*} M$ stays in the Darboux neighbourhood and hence the trajectories that define the "global" Floer differential are those that were already counted in the local differential.

The end of the proof is similar to [5]. The Novikov ring which defines the Novikov homology associated to $f^{\star} u$ and the covering $\bar{L} \rightarrow L$ is $\Lambda_{u}$, so that the Morse complex above is exactly $C_{\star}(\bar{L} \rightarrow L, h, \xi)$. The $\Lambda_{u}$-complexes

$$
\Lambda_{u} \otimes_{\Lambda} C_{\star}\left(L, \varphi_{t}^{d g+d H_{t}}, J_{t}\right) \text { and } \Lambda_{u} \otimes_{\Lambda} C_{\star}(\bar{L} \rightarrow L, h, \xi)
$$

are homotopy equivalent, so that the homologies

$$
F H(\bar{L}, \sigma u) \simeq H(\bar{L}, u)
$$

are isomorphic.

Propositions 4.1 and 4.5 imply that the set

$$
\{\sigma \in] 0 ;+\infty\left[\mid F H(\bar{L}, u) \simeq H_{\star}\left(\bar{L}, f^{\star} u\right)\right\}
$$

is nonempty, open and closed, hence equal to $] 0 ;+\infty[$ so that we have proved the following theorem:

Theorem 4.6. Let $u$ be an element of $H^{1}(M)$.

Assume that the Lagrangian submanifold $L$ is monotone on the loops and its Maslov number $N_{L}$ satisfies

$$
N_{L} \geq \operatorname{dim}(M)+2 .
$$

Then the Floer homology $F H(\bar{L}, u)$ is isomorphic to the Novikov homology $H\left(\bar{L}, f^{\star} u\right)$. 
If we only assume that $N_{L} \geq 2$, we do not necessarily have an isomorphism between the Floer homology and the Novikov homology of $L$. This is also the case in usual Floer theory, but we have the spectral sequence described by Biran in 33 to relate it to the singular homology of the Lagrangian submanifold. We can also define in the monotone case a spectral sequence whose first page is the Novikov homology of $L$ and that converges to the Floer-Novikov homology. The following theorem gives a precise description of this spectral sequence.

Let $\bar{A}$ be the $\Lambda_{u}$-module $\bar{A}=\Lambda_{u}\left[T, T^{-1}\right]$ of Laurent polynomials with coefficients in $\Lambda_{u}$. Let $\tau_{i}: \bar{A} \rightarrow \bar{A}$ be the multiplication by $T^{i}$. We define the degree of $T$ to be $N_{L}$. Then

$$
\bar{A}=\bigoplus_{i \in \mathbb{Z}} \bar{A}^{i}
$$

where $\bar{A}^{i}=\Lambda_{u} T^{i / N_{L}}$ if $i \equiv 0 \bmod N_{L}$ and $\bar{A}^{i}=\{0\}$ otherwise.

Theorem 4.7. There exists a spectral sequence $\left\{E_{r}^{p, q}, d_{r}\right\}$ satisfying the following properties:

(1) $E_{0}^{p, q}=C_{p+q-p N_{L}}\left(\bar{L}, f^{\star} u\right) \otimes \bar{A}^{p N_{L}}$ and $d_{0}=\partial_{0} \otimes 1$;

(2) $E_{1}^{p, q}=H_{p+q-p N_{L}}\left(\bar{L}, f^{\star} u\right) \otimes \bar{A}^{p N_{L}}$ and $d_{1}=\left[\partial_{1}\right] \otimes \tau$ where

$$
\left[\partial_{1}\right]: H_{p+q-p N_{L}}\left(\bar{L}, f^{\star} u\right) \longrightarrow H_{p+1+q-(p+1) N_{L}}\left(\bar{L}, f^{\star} u\right)
$$

is induced by $\partial_{1}$;

(3) For all $r \geq 1, E_{r}^{p, q}$ can be written $E_{r}^{p, q}=V_{r}^{p, q} \otimes \bar{A}^{p N_{L}}$ with $d_{r}=\delta_{r} \otimes \tau_{r}$, $V_{r}^{p, q}$ are modules on $\Lambda_{u}, \delta_{r}: V_{r}^{p, q} \rightarrow V_{r}^{p+r, q-r+1}$ are morphisms and satisfy $\delta_{r} \circ \delta_{r}=0$. Moreover,

$$
V_{r+1}^{p, q}=\frac{\operatorname{ker}\left(\delta_{r}: V_{r}^{p, q} \rightarrow V_{r}^{p+r, q-r+1}\right)}{\operatorname{Im}\left(\delta_{r}: V_{r}^{p-r, q+r-1} \rightarrow V_{r}^{p, q}\right)} ;
$$

(4) $\left\{E_{r}^{p, q}, d_{r}\right\}$ collapses at page $\kappa+1$, where $\kappa=\left[\frac{\operatorname{dim}(L)+1}{N_{L}}\right]$ and the spectral sequence converges to $F H(\bar{L}, u)$, i.e.

$$
\bigoplus_{p+q=\ell} E_{\infty}^{p, q} \cong F H^{\ell\left(\bmod N_{L}\right)}(\bar{L}, u)
$$

(5) For all $p \in \mathbb{Z}, \bigoplus_{q \in \mathbb{Z}} E_{\infty}^{p, q} \cong F H(\bar{L}, u)$.

Proof. In order to describe the spectral sequence, we look at the proof of Proposition 4.5 and the Hamiltonian $\mathcal{H}$ defined with the $C^{2}$-small function $h$. We suppose, as in [3], that $h$ has exactly one relative minimum $x_{0}$ and we use $x_{0}$ as base point for the Floer complex so that we can fix the grading by $\mathbb{Z} / N_{L}$. As we have the decomposition (see [18):

$$
C_{i\left(\bmod N_{L}\right)}\left(\bar{L}, \varphi_{t}^{H_{t}^{0}}, J_{t}^{0}\right)=\bigoplus_{j \equiv i\left(\bmod N_{L}\right)} C_{j}\left(\bar{L}, f^{\star} u\right) .
$$

we can decompose the differential

$$
\partial: C_{\star} \bmod N_{L}\left(\bar{L}, \varphi_{t}^{H_{t}^{0}}, J_{t}^{0}\right) \longrightarrow C_{\star+1 \bmod N_{L}}\left(\bar{L}, \varphi_{t}^{H_{t}^{0}}, J_{t}^{0}\right)
$$


in $\partial=\sum_{j \in \mathbb{Z}} \partial_{j}$ with

$$
\partial_{j}: C_{\star}\left(\bar{L}, f^{\star} u\right) \rightarrow C_{\star+1-j N_{L}}\left(\bar{L}, f^{\star} u\right) .
$$

Moreover, by the index computations of [18],

$$
\partial_{j}=0 \text { if } j<0 \text { or } j>\kappa=\left[\frac{\operatorname{dim} L+1}{N_{L}}\right],
$$

so that

$$
\partial=\partial_{0}+\cdots+\partial_{\kappa} .
$$

The differential $\partial_{0}$ counts the trajectories that stay in the neighbourhood $\mathcal{U}$, it corresponds to the differential of the local Floer homology and as before, the homology of the complex $\left(C_{\star}\left(\bar{L}, f^{\star} u\right), \partial_{0}\right)$ can be identified to the Novikov homology $H\left(\bar{L}, f^{\star} u\right)$. The other operators $\partial_{1}, \ldots, \partial_{\kappa}$ count the trajectories that leave the neighbourhood $\mathcal{U}$.

In order to define and prove the properties of the spectral sequence, it suffices to substitute the coefficients in $\mathbb{Z} / 2$ in the proof of [3, Theorem 5.2] by coefficients in $\Lambda_{u}$.

Remark 4.8. Note that if $N_{L} \geq \operatorname{dim}(M)+2$, the spectral sequence collapses at page $\kappa+1=1$ and we recover Theorem 4.6 .

\subsection{Proof of Theorems 1.4 and 1.5}

Proof of Theorem 1.4. As in [5, Section 4.1], it is a consequence of the proofs of Propositions 1.1, 4.5 and Theorem 4.7. Thanks to Proposition 1.4, we can define for $\sigma$ small enough a lift of the morphism of $\Lambda_{f^{\star} u}$-complex $\Gamma_{\star}$ between $C_{\star}\left(L, \varphi_{t}^{\alpha+d H_{t}}, J_{t}\right)$ and $C_{\star}\left(L, \varphi_{t}^{\alpha+\sigma \eta+d H_{t}}, J_{t}^{\prime}\right)$ which induces an isomorphism in homology:

$$
F H(L, u) \simeq F H(L,(1+\sigma) u) .
$$

In order to relate $F H(L, \sigma u)$ and $H_{\star}\left(L, f^{\star} u\right)$, we use a lift of the morphism $\Gamma_{\star}^{0}$ defined in the proof of Proposition 4.5 and a spectral sequence analogous to the one in Theorem 4.7 with the first page expressed in terms of $H_{\star}\left(L, f^{\star} u\right)$ and the $\Lambda_{f^{\star} u}$-module $A=\Lambda_{f^{\star} u}\left[T, T^{-1}\right]$.

When $M$ is the total space of a fibration on the circle, Theorem 1.4 enables us to prove Theorem 1.5 in the case where $f_{\star}: \pi_{1}(L) \longrightarrow \pi_{1}(M)$ is surjective. Thanks to Remark 3.6, this will be enough to prove the theorem under the hypothesis $N_{L} \geq \operatorname{dim}(M)+1$ or $\left(N_{L}=\operatorname{dim}(M)\right.$ and $\left[\pi_{1}(M): \pi_{1}(L)\right]$ is finite).

Proof of Theorem 1.5 in the case $N_{L} \geq \operatorname{dim}(M)+1$. Since the manifold $M$ is the total space of a fibration on the circle, there exists a closed one-form $\alpha$ that does not vanish on $M$. Consider the symplectic isotopy $\varphi_{t}$ of $T^{*} M$ spanned by $X^{\alpha}$ :

$$
\varphi_{t}(p, q)=\left(p+t \alpha_{q}, q\right) .
$$

For $T$ large enough, $\varphi_{T}(L) \cap L=\emptyset$ and without restricting generality we can assume that $T=1$. Then the Floer complex defined in Section 3 is empty and the Floer homology $F H(L, u)$ is trivial. 
But, as $u \neq 0$ and as $f_{\star}: \pi_{1}(L) \rightarrow \pi_{1}(M)$ is surjective, $f^{\star} u \neq 0$ and by Proposition 2.5, if the presentation of the fundamental group of $L$ satisfies $p-q \geq 2$ or if the fundamental group is a free product of two non trivial groups, then $H_{1}\left(L, f^{\star} u\right) \neq 0$.

If $N_{L} \geq \operatorname{dim}(M)+2$, then by Theorem 1.4 the Floer homology of $L$ is isomorphic to the Novikov homology $H_{\star}\left(L, f^{\star} u\right)$ and this contradicts $H_{1}\left(L, f^{\star} u\right) \neq 0$. If $N_{L}=\operatorname{dim}(M)+1 \geq 2$, then the spectral sequence defined in Theorem 4.7 collapses at page $\kappa+1=2$ and converges to the Floer homology of $L$. But for $p=0$ and $q=1$,

$$
\left[\partial_{1}\right]: H_{1}\left(L, f^{\star} u\right) \longrightarrow H_{2-N_{L}}\left(L, f^{\star} u\right)=\{0\}
$$

and

$$
\left[\partial_{1}\right]: H_{N_{L}}\left(L, f^{\star} u\right)=\{0\} \longrightarrow H_{1}\left(L, f^{\star} u\right),
$$

hence $E_{0,1}^{2}=H_{1}\left(L, f^{\star} u\right)$. We get also a contradiction in this case.

Proof of Theorem 1.5 in the case $N_{L} \geq \operatorname{dim}(M)$. We can even extend the result to the case $N_{L}=\operatorname{dim}(M)$ thanks to a property of the Novikov homology $H_{\star}\left(L, f^{\star} u\right.$ ) (see Latour 11] or Levitt [13): since $f^{\star} u \neq 0$, there exists in the class of $f^{\star} u$ a one-form $\alpha^{\prime}$ which has no critical point of index 0 or $\operatorname{dim}(M)$. In particular, the groups $H_{0}\left(L, f^{\star} u\right)$ and $H_{\operatorname{dim}(M)}\left(L, f^{\star} u\right)$ are trivial.

By Theorem 1.4, there exists a spectral sequence converging to the Floer homology of $L$ and whose first page can be described with the Novikov homology of $L$. In the case $N_{L}=\operatorname{dim}(M) \geq 2$, the spectral sequence collapses at the second page,

$$
\left[\partial_{1}\right]: H_{1}\left(L, f^{\star} u\right) \longrightarrow H_{2-N_{L}}\left(L, f^{\star} u\right)=\{0\}
$$

and

$$
\left[\partial_{1}\right]: H_{N_{L}}\left(L, f^{\star} u\right)=\{0\} \longrightarrow H_{1}\left(L, f^{\star} u\right),
$$

so that $E_{0,1}^{2}=H_{1}\left(L, f^{\star} u\right)$. As before, this leads to a contradiction.

\section{Appendix: Proof of Lemma [3.7}

(i) Assume first that $\bar{L}$ is path-connected. Choose a base point $\ell$ in $L$ and let $m=i(\ell)$ be its image in $T^{*} M$. Choose also a lift $\tilde{\ell}$ of $\ell$ in $\bar{L}$. Let $g$ be an element of $\pi_{1}(M)$. As $\bar{L}$ is path-connected, there exists a path $\widetilde{\gamma}$ from $\tilde{\ell}$ to $\widetilde{\ell}^{g}$ in $\bar{L}$. The image $(\pi \circ \widetilde{p} \circ \widetilde{\imath})(\widetilde{\gamma})$ of that path in $M$ is a loop representing $g$ and its image in $L$ is thus a loop $\gamma$ such that $f_{\star}([\gamma])=g$.

Conversely, assume that the map $\pi_{1}(L) \rightarrow \pi_{1}(M)$ is surjective. As $L$ is supposed to be path-connected, to prove that $\bar{L}$ is path-connected, it is enough to prove that two points in the same fiber can be joined by a path in $\bar{L}$. Let $\widetilde{\ell}_{1}$ and $\widetilde{\ell}_{2}$ two elements of $\bar{L}$ in the fiber of the point $\ell$ of $L$. The two points $\widetilde{\imath}\left(\widetilde{\ell}_{1}\right)$ and $\widetilde{\imath}\left(\widetilde{\ell}_{2}\right)$ of $T^{*} \widetilde{M}$ can be joined in $T^{*} \widetilde{M}$ by a path which projection on $T^{*} M$ is a loop $c$ such that $\widetilde{\imath}\left(\tilde{\ell}_{2}\right)=\left(\widetilde{\imath}\left(\tilde{\ell}_{1}\right)\right)^{[c]}$. But by assumption, the element $[c]$ of the group $\pi_{1}(M)$ has an antecedent in $\pi_{1}(L)$. Let $\gamma$ be a loop in $L$ based in $\ell$ representing this antecedent. If 
$\widetilde{\gamma}$ is the lift with starting point $\widetilde{\ell}_{1}$ of $\gamma$ in $\bar{L}$, its endpoint $\tilde{\ell}_{1}^{[\gamma]}$ must have $\left(\widetilde{\imath}\left(\tilde{\ell}_{1}\right)\right)^{[c]}$ as image by $\widetilde{\imath}$, and this proves that $\widetilde{\gamma}$ is a path from $\tilde{\ell}_{1}$ to $\tilde{\ell}_{2}$.

(ii) By definition, $\bar{L}$ fits into the following commutative diagram:

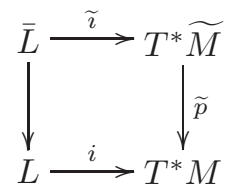

As $T^{*} \widetilde{M}$ is simply connected, the diagram induced on the fundamental groups gives that $\operatorname{Im}\left(\pi_{1}(\bar{L}) \rightarrow \pi_{1}(L)\right)$ is included in $K$. Conversely, any loop of $L$ whose image in $M$ is homotopic to the constant path can be lifted to $T^{*} \widetilde{M}$ in a loop of $\bar{L}$.

(iii) The embedding is monotone because the symplectic structure (and the Liouville form) on $T^{*} \widetilde{M}$ are obtained by taking the pull-back of those of $T^{*} M$.

\section{References}

[1] V. I. Arnol'd. The first steps of symplectic topology. Uspekhi Mat. Nauk, 41(6(252)):3-18, 229, 1986.

[2] M. Audin. Fibrés normaux d'immersions en dimension double, points doubles d'immersions lagrangiennes et plongements totalement réels. Comment. Math. Helv., 63(4):593-623, 1988.

[3] P. Biran. Lagrangian non-intersections. Geom. Funct. Anal., 16(2):279326, 2006.

[4] L. Buhovski. Homology of Lagrangian submanifolds in Cotangent Bundles. arXiv:math/0312265.

[5] M. Damian. Constraints on exact Lagrangians in cotangent bundles of manifolds fibered over the circle. arXiv:0710.0511, to be published in Commentarii Mathematici Helvetici.

[6] A. Floer. Morse theory for Lagrangian intersections. J. Differential Geom., 28(3):513-547, 1988.

[7] A. Floer. The unregularized gradient flow of the symplectic action. Comm. Pure Appl. Math., 41(6):775-813, 1988.

[8] K. Fukaya, P. Seidel, and I. Smith. Exact Lagrangian submanifolds in simply-connected cotangent bundles. Invent. Math., 172(1):1-27, 2008.

[9] R. Hind. Lagrangian spheres in $S^{2} \times S^{2}$. Geom. Funct. Anal., 14(2):303318, 2004.

[10] F. Lalonde and J.-C. Sikorav. Sous-variétés lagrangiennes et lagrangiennes exactes des fibrés cotangents. Comment. Math. Helv., 66(1):18-33, 1991. 
[11] F. Latour. Existence de 1-formes fermées non singulières dans une classe de cohomologie de de Rham. Inst. Hautes Études Sci. Publ. Math., (80):135194, 1994.

[12] H. V. Lê and K. Ono. Symplectic fixed points, the Calabi invariant and Novikov homology. Topology, 34(1):155-176, 1995.

[13] G. Levitt. 1-formes fermées singulières et groupe fondamental. Invent. Math., 88(3):635-667, 1987.

[14] D. Nadler. Microlocal branes are constructible sheaves. arXiv:math/0612399.

[15] S. P. Novikov. Multivalued functions and functionals. An analogue of the Morse theory. Dokl. Akad. Nauk SSSR, 260(1):31-35, 1981.

[16] Y.-G. Oh. Floer cohomology of Lagrangian intersections and pseudoholomorphic disks. I. Comm. Pure Appl. Math., 46(7):949-993, 1993.

[17] Y.-G. Oh. Addendum to: Floer cohomology of Lagrangian intersections and pseudo-holomorphic disks. I. Comm. Pure Appl. Math., 48(11):1299 $1302,1995$.

[18] Y.-G. Oh. Floer cohomology, spectral sequences, and the Maslov class of Lagrangian embeddings. Internat. Math. Res. Notices, (7):305-346, 1996.

[19] A. V. Pazhitnov. Surgery on the Novikov complex. K-Theory, 10(4):323$412,1996$.

[20] L. Polterovich. Monotone Lagrange submanifolds of linear spaces and the Maslov class in cotangent bundles. Math. Z., 207(2):217-222, 1991.

[21] A. F. Ritter. Novikov-symplectic cohomology and exact Lagrangian embeddings. arXiv:0711.1396.

[22] J.-C. Sikorav. Homologie de Novikov associe une classe de cohomologie relle de degr 1. Thse Orsay, 1987.

[23] C. Viterbo. Intersection de sous-variétés lagrangiennes, fonctionnelles d'action et indice des systèmes hamiltoniens. Bull. Soc. Math. France, 115(3):361-390, 1987.

[24] C. Viterbo. Functors and computations in Floer homology with applications. I. Geom. Funct. Anal., 9(5):985-1033, 1999.

Institut de recherche mathmatique avance, Universit Louis Pasteur 7, rue Ren Descartes, 67084 Strasbourg, France.

e-mail address: gadbled@math.u-strasbg.fr 